\title{
Crocetin suppresses angiogenesis through inhibiting Sonic hedgehog signaling pathway in gastric cancer
}

\section{Ye Zhou ( $\nabla$ zhouye79@yahoo.com )}

Fudan University Shanghai Cancer Center

\section{Mingde Zang}

Fudan University Shanghai Cancer Center

\section{Junyi Hou}

Shanghai Jiao Tong university school of medicine, Ruijin hospital

\section{Jiangli Wang}

fudan university shagnhai cancer center

\section{Yakai Huang}

Fudan University Shanghai Cancer Center

\section{Xusheng Ding}

Shanghai Jiao Tong University school of Medicine, Ruijin hospital.

\section{Yanong Wang}

Fudan University Shanghai Cancer Center

\section{Research}

Keywords: GC, Crocetin, Angiogenesis, Shh

Posted Date: October 7th, 2020

DOl: https://doi.org/10.21203/rs.3.rs-86049/v1

License: (c) (1) This work is licensed under a Creative Commons Attribution 4.0 International License. Read Full License 


\section{Abstract}

\section{Background}

Crocetin is an active component of saffron stigma, which has important therapeutic effects on various diseases including tumors, arthritis, hemorrhages, etc. However, the effects of crocetin on gastric cancer (GC) cells and their underlying mechanisms remain unclear.

\section{Methods}

Cell counting kit-8 (CCK-8), transwell assays, F-actin staining, tube formation and vasculogenic mimicry (VM) assays were used to examine the effects of crocetin on cell proliferation, migration and angiogenesis in GC. Enzyme linked immunosorbent assay (ELISA) and Western blot assay were performed to evaluate expression level of Sonic hedgehog (Shh) signaling and activation of epithelial-mesenchymal transition (EMT) in GC cells treated with or without crocetin. Proliferation of xenograft tumors was detected by immunohistochemistry.

\section{Results}

Crocetin significantly inhibited tube formation of human umbilical vein endothelial (HUVEC) cells and VM formation of GC cells, as well as its proliferation, invasion and migration. Crocetin destroyed cytoskeleton and mosaic vessels formed by HUVEC and GC cells. Furthermore, we found that crocetin suppressed cell proliferation, migration, EMT, tube and VM formation through inhibiting Shh signaling pathway. Utilization of recombinant Shh reversed the suppressing effects of crocetin on cell proliferation, migration, angiogenesis and EMT. In addition, anti-tumor effect of crocetin was confirmed in xenograft tumors.

\section{Conclusions}

Crocetin suppressed GC progression by inhibiting cell proliferation, migration and angiogenesis including tubes formed by HUVEC cells and VM vessels formed by GC cells, which were mediated by suppressing Shh signaling pathway. These results indicated that crocetin may function as an effective therapeutic drug against GC.

\section{Background}

Gastric cancer (GC) is the fifth most common cancer and the third major cause of cancer-related deaths worldwide because of its highly metastatic properties and rapid progression to advanced stages [1, 2]. The only curative therapy for localized disease is radical surgery, but most patients are at advanced stages at the time of diagnosis [3]. Chemoresistance is another crucial obstacle for GC treatment, particularly in advanced GC [4]. Therefore, it is of great significance and necessity to clarify mechanisms underlying GC progression, facilitating the development of new drugs and therapeutic strategies targeting GC. 
Phytochemicals derived from plants are considered as good sources for developing anticancer drugs. Among these phytochemicals, saffron shows important therapeutic effects on various diseases for many centuries, which has been attributed mostly to crocetin recently $[5,6]$. As an active constituent of saffron, crocetin functions in different mechanisms including inhibition of pro-inflammatory mediators, prevention of proliferation or stimulation of apoptosis in cancer cells [7-9]. Although there is no direct data about the $L_{5}$ value of crocetin, a clinical trial conducted to evaluate the safety of crocetin has shown that excessive intake of crocetin $(37.5 \mathrm{mg} /$ day for four weeks) produces no significantly clinical changes or side effects in any healthy adult volunteers [10]. Considering that crocetin is tolerable without major toxicity at therapeutic dosage and shows great anti-tumor activity, it may be an effective drug for the treatment of GC.

Tumor angiogenesis is an important cancer hallmark playing a key role in tumor progression, including rapid growth, relapse and metastasis $[11,12]$. However, angiogenesis is not the unique way to provide nutrients and oxygen for tumor continuous growth $[13,14]$, various malignant tumors obtain nutrients via organizing themselves into vascular-like structures called vasculogenic mimicry (VM), which takes place independently of angiogenesis or endothelial cells $[15,16]$. Recently, accumulated research has reported the existence of VM in a variety of malignant tumors, such as melanoma [17], hepatocellular carcinoma [18], breast cancer [19], prostate cancer [20], lung cancer [21], colorectal cancer [22] and GC [1]. Moreover, VM is significantly correlated with poor prognosis of cancer patients [24] and has emerged as a new target for anti-tumor therapy [25]. Although there is no lack of research on signaling pathways associated with VM in highly aggressive malignant tumors [26], the role of Shh/Gli1 signaling in VM formation is rarely discussed.

As a new recognized pro-angiogenic factor, Sonic hedgehog (Shh) binds to its receptor Patched (PTCH) and then induces release of smoothened (SMO), which mediates downstream activation of Gli1, regulating various target genes involved in cell growth and angiogenesis [27, 28]. Activation of Shh/Gli1 signaling pathway has been observed during cancer angiogenesis process [29] and several inhibitors targeting Shh/Gli1 pathway show suppressing effects on tumor growth and angiogenesis [30, 31]. Therefore, we designed this research to clarify the effects of crocetin on angiogenesis and VM formation in GC, and to explore its potential targeting signaling, such as Shh/Gli1 pathway.

In this study, we investigated the role of crocetin in GC processes, including angiogenesis, migration and invasion, which were in addition to previous researches only limited to illustrating the anti-proliferation and pro-apoptosis effects of crocetin on GC cells. As a result, we found that crocetin not only suppressed angiogenesis of HUVECs, but also inhibited VM formation, as well as cell proliferation and metastasis of GC cells, and these inhibitory effects of crocetin were achieved by inhibiting activation of Shh/Gli1 signaling pathway. Thus, our study revealed that crocetin plays an anti-tumor role in GC angiogenesis and metastasis, providing an experimental basis for further investigation of crocetin in the treatment against GC.

\section{Methods}




\section{Cell lines}

The human GC cell line NCl-N87 was maintained in our lab while Hs-746T was purchased from American Type Culture Collection, and human umbilical vein endothelial (HUVEC) cells were purchased from Shanghai Institutes for Biological Sciences, Chinese Academy of Sciences. Cells were cultured at $37^{\circ} \mathrm{C}$ in $5 \% \mathrm{CO}_{2}$ and saturation humidity in RPMI- 1640 medium with $10 \%$ fetal bovine serum containing penicillin and streptomycin.

\section{Endothelial tube formation}

HUVEC cells were plated in 96-well plate coated with $50 \mu$ matrigel (BD Bioscience, CA, USA) at the density of $3 \times 10^{4}$ cells/well and treated with or without different concentrations of crocetin. Tubes were photographed by microscopy and evaluated by Image Pro Plus software.

\section{Cell proliferation assay}

Cell proliferation was monitored by Cell Counting Kit-8 (CCK-8). HUVEC cells or GC cells plated in 96-well plate at the concentration 2000 cells/well were treated with or without crocetin. Cell proliferation was measured every $24 \mathrm{~h}$ for 5 days after adding CCK-8 $2 \mathrm{~h}$ at the absorbance $450 \mathrm{~nm}$ using Epoch Microplate Spectrophotometer (Bio Tek).

\section{Migration and invasion assays}

A number of $1 \times 10^{5}$ HUVEC cells or GC cells were suspended in serum-free RPMI-1640 medium containing crocetin or not and plated in transwell upper chamber $(8 \mu \mathrm{m}$ for 24 -well plate; Corning Costar, NY, USA) coated with or without Matrigel (BD Bioscience, CA, USA) for migration or invasion assay respectively. Cells were fixed by $10 \%$ formalin and stained with $0.5 \%$ crystal violet after $24 \mathrm{~h}$ incubation in 24-well plates. Finally, cells in the lower chamber were photographed and counted by inverted microscopy.

\section{Filamentous actin (F-actin) staining}

HUVEC cells and GC cells were plated into 8-well glass (Merck Millipore) and treated with or without crocetin for $24 \mathrm{~h}$, and then fixed using $4 \%$ freshly made paraformaldehyde for 10 minutes. After permeabilization with $0.2 \%$ Triton X-100, the cells were blocked using $3 \%$ bovine serum albumin for 30 minutes. Next, the cells were stained with rhodamine phalloidin (1:20; CST) to visualize the cytoskeleton while the nuclei were stained with DAPI. Slides were imaged and analyzed on a fluorescence microscope.

\section{Vasculogenic mimicry (VM) formation assay}

Serum-free RPMI-1640 medium and Matrigel were mixed according to 2:1. The mixture was then seeded onto $16 \mathrm{~mm}$ glass cover slides in 6-well plate and was allowed to polymerize for $2 \mathrm{~h}$ at $37^{\circ} \mathrm{C}$. Then $1 \times$ 
$10^{5} \mathrm{GC}$ cells were seeded onto each slide and cultured for 3 days at $37^{\circ} \mathrm{C}$ with $5 \% \mathrm{CO}_{2}$. Cells then were fixed by $4 \%$ paraformaldehyde and stained by PAS stain according to the manufacturer's protocols.

After counterstained with hematoxylin, slides were dehydrated and covered.

\section{Three-dimensional (3D) culture and mosaic vessel assays}

A 24-well plate was coated with $100 \mu \mathrm{l}$ Matrigel/well and then was allowed to polymerize for $2 \mathrm{~h}$ at $37^{\circ} \mathrm{C}$. Hs-746T GC cells labeled with EGFP and HUVEC cells without EGFP label were mixed according to 1:1. Next, mixed cells were plated into 24-well at the density of $1 \times 10^{5}$ cells/well and cultured in complete growth medium with or without crocetin. Tubes were photographed by an AMG fluorescence microscope after $24 \mathrm{~h}$ incubation at $37{ }^{\circ} \mathrm{C}$ with $5 \% \mathrm{CO}_{2}$ and were evaluated by Image Pro Plus software.

\section{Quantitative Real-time PCR (RT-qPCR)}

RNA was extracted from GC cells treated with or without crocetin, and then RNA was reversed to cDNA. RT-qPCR was performed using SYBR-green according to manufacturer's instructions. Primers for HIF-1a, forward 5'-GAACGTCGAAAAGAAAAGTCTCG-3', reverse 5'- CCTTATCAAGATGCGAACTCACA-3'; Notch-1, forward 5'-GCTTGTGGTAGCAAGGAAGC-3', reverse 5'-CCACATTCAAGTGGCTGATG-3'; Shh, forward 5'CTGCTCGGTGAAAGCAGAGA-3', reverse 5'- CGCGTCTCGATCACGTAGAA-3'; VEGF, forward 5'AGGGCAGAATCATCACGAAGT-3', reverse 5'-AGGGTCTCGATTGGATGGCA-3'; GAPDH, forward 5'GGACCTGACCTGCCGTCTAG-3', reverse 5'-GTAGCCCAGGATGCCCTTGA-3'.

\section{Western blotting}

The method was consistent with the previous[32]. In brief, proteins of cells were separated by SDS-PAGE and then transferred into PVDF membranes. Primary antibody (1:1000 dilutions) Shh, PTCH2, Sufu, GLi1, E-cadherin, $\beta$-catenin, vimentin were purchased from cell signaling technology (CST, USA). GAPDH (1:10000 dilutions) was purchased from Proteintech. After incubation with primary antibody, secondary antibody followed. Finally, the results were visualized by Tanon system.

\section{Enzyme linked immunosorbent assay (ELISA)}

Concentrations of Shh secreted from HUVEC cells and Hs-746T GC cells induced by crocetin were assayed by ELISA according to the manufacturer's instructions. The absorbance at OD $450 \mathrm{~nm}$ was read and the level of Shh expression were calculated according to the standard curve.

\section{Nude mice tumorigenesis and immunohistochemistry}

Hs-746T GC cells $\left(1 \times 10^{6}\right.$ cells) were subcutaneously injected into 4-week-old male BALB/c nude mice (Institute of Zoology, China Academy of Sciences) to evaluate the role of crocetin in tumor growth in vivo. Tumor nodules were measured every week and were calculated using the formula: tumor volume $=$ 
(Width ${ }^{2} \times$ Length) / 2. Mice were killed 4 weeks after injection. Tumors were weighed and fixed for immunohistochemistry staining $(\mathrm{IHC})$.

For IHC, sections staining was performed according to the DAKO protocol using primary antibody (1:200 dilutions) E-cadherin, vimentin and Ki-67.

\section{Statistical analysis}

Data are shown as mean \pm SD. Differences between experimental groups were assessed by the Student's $t$ test or one-way ANOVA. A two-tailed value of $P<0.05$ was considered statistically significant. Statistical analysis was performed using IBM SPSS 19.0 software (SPSS Inc).

\section{Results}

\section{Crocetin suppresses tube formation of HUVEC cells as well as cell proliferation and migration ability}

HUVEC cells were plated in 96-well plate coated with matrigel and cultured with $0,50,100,200 \mu \mathrm{M}$ crocetin for $12 \mathrm{~h}$ at $37^{\circ} \mathrm{C}$ with $5 \% \mathrm{CO}_{2}$. Tube formation ability of HUVEC cells were detected by counting the number of tubes and junctions. As shown in Fig. 1a, the control group exhibited apparently tubes formation while that of the groups treated with 100 and $200 \mu \mathrm{M}$ crocetin were significantly inhibited (Fig. $1 b, c)$. Next, we tested the effects of crocetin on HUVEC cell proliferation and migration, which were known to be correlated with tube formation of HUVEC cells. CCK-8 assay was performed every $24 \mathrm{~h}$ and results showed that crocetin treatment effectively inhibited HUVEC cell proliferation in a dose-dependent manner (Fig. 1d). Similarly, the migration ability of HUVEC cells was also suppressed after crocetin treatment (Fig. 1e). Moreover, the cytoskeleton of HUVEC cells treated with crocetin was broken as evidenced by F-actin staining (Fig. 1f). These findings indicate that crocetin can inhibit tube formation of HUVEC cells through reducing cell proliferation and motility.

\section{Crocetin suppresses VM and mosaic vessels formation}

Different from blood vessels formed by HUVEC cells, vessel-like structure defined as vasculogenic mimicry (VM) is formed by malignant tumor cells. According to our results, less VM structures formed by $\mathrm{NCl}-\mathrm{N} 87$ and Hs-746T GC cells were observed in crocetin-treated group than control group (Fig. 2a). And the VM forming ability was evaluated by the number of tubes (Fig. 2b) and the number of intersections (Fig. 2c). In addition, 3D culture assay using periodic acid-Schiff (PAS) staining showed crocetin obviously reduced VM formation in GC cells (Fig. 2d), which was evidenced by calculating the number of tubes (Fig. 2e) and junctions (Fig. 2f).

Mosaic vessels, which serve as the bridge to transfer nutrition for HUVEC and VM vessels, are of great importance for tumor growth. To verify whether crocetin affects formation of mosaic vessels in vitro, Hs746T GC cells were mixed with HUVEC cells according to the proportion 1:1 at the total number $1 \times 10^{5}$, then the mixture cells were plated into 24 -well plate which was previously coated with Matrigel and then 
cultured with or without crocetin. GC cells labeled with EGFP showed green image while HUVEC cells without EGFP showed gray under the fluorescence microscope (Fig. $2 \mathrm{~g}$ ). As shown, mosaic vessels were destroyed in crocetin treatment group (Fig. $2 \mathrm{~g}$ ) and crocetin treatment effectively decreased the number of mosaic vessels intersections (Fig. 2h). These results suggest crocetin may serve as a functional drug against GC.

\section{Crocetin inhibits motility and proliferation of GC cells}

More experiments were performed to determine the role of crocetin in inhibiting the pathological process of GC. Shrank cytoskeleton of NCl-N87 (Fig. 3a) and Hs-746T (Fig. 3b) cells were observed in the existence of crocetin, indicating that crocetin can suppress the motility of GC cells. Similarly, crocetin treatment showed significantly inhibitory effects on GC cells migration (Fig. 3c, d) and invasion (Fig. 3e, f) ability according to transwell assays. Furthermore, CCK-8 assay showed that crocetin inhibited cell proliferation of NCl-N87 (Fig. 3g) and Hs-746T (Fig. 3h) in a dose-dependent manner.

\section{Crocetin suppresses Shh signaling pathway}

In order to determine the mechanism underlying the anti-tumor effects of crocetin, we first detected RNA levels of some signaling pathways related to angiogenesis by quantitative RT-PCR. We found that expression of Shh and VEGF were markedly downregulated due to crocetin treatment while HIF-1a and Notch1 did not show significant differences (Fig. 4a). The same results were obtained from ELISA experiment, namely that crocetin decreased the protein level of Shh both in Hs-746T GC cells and HUVEC cells (Fig. 4c). In addition, expression of downstream target genes involved in Shh signaling pathway were also inhibited after crocetin treatment demonstrated by WB, such as PTCH2, SUFU and Gli1 (Fig. 4b). According to further studies, we found that crocetin suppressed migration of HUVECs induced by Hs746T (Fig. 4d, e), and in the same way that crocetin inhibited HUVECs-induced migration of Hs-746T (Fig. $4 f, g)$. These findings suggest that crocetin affect GC progression via suppressing Shh signaling pathway.

\section{Recombinant Shh reverses inhibitory effects of crocetin on EMT and GC progression}

The remodeling of the cytoskeleton upon crocetin treatment indicated that crocetin may regulate this process by inhibiting EMT. We observed that the epithelial biomarker E-cadherin was increased while the mesenchymal biomarker vimentin, as well as $\beta$-catenin was reduced both in HUVECs and Hs-746T cells upon crocetin treatment (Fig. 5a). However, the inhibitory effect of crocetin on EMT was reversed by administration of recombinant Shh (Fig. 5b). In addition, cell proliferation (Fig. 5c) and migration of HUVECs (Fig. 5d) and Hs-746T cells (Fig. 5e) suppressed by crocetin were recovered following adding recombinant Shh. Moreover, Shh promoted tube formation of HUVEC and VM formation of GC cells in the absence of crocetin (Fig. 5f), which were obviously shown by evaluation of the number of tubes (Fig. $5 \mathrm{~g}$ ) and junctions (Fig. 5h). These observations confirm that crocetin can suppress GC progression by inhibiting Shh signaling. 


\section{Crocetin inhibits tumor growth in vivo}

To clarify the effects of crocetin on tumor growth in vivo, Hs-746T cells were injected subcutaneously into nude mice. Then nude mice forming tumors were injected intraperitoneally with PBS or crocetin (10 $\mathrm{mg} / \mathrm{kg})$ for 6 times. We found that the tumor weight $(P<0.05)$ and tumor volume $(P<0.05)$ in crocetintreated mice were less than that in the control group (Fig. 6a-C). Furthermore, E-cadherin, vimentin and Ki67 expression were decreased in tumors from crocetin-treated mice (Fig. 6d), which were consistent with the results found in vitro. These findings illustrate that crocetin exerts a suppressed effect on GC growth in vivo.

\section{Discussion}

GC is one of the most aggressive malignancies worldwide. Despite improvements in early diagnosis and multi-agent chemotherapy, the overall prognosis of GC patients remains poor, especially those diagnosed with metastatic GC [33]. The main available chemotherapeutic agents for GC can hardly achieve the desired therapeutic effects, which is to some extent because of drug resistance resulting from genetic heterogeneity among tumor cells and individual variations among patients [34, 35]. Up to now, numerous phytochemicals exhibit anti-cancer properties, some of which such as taxol, Vinca alkaloids, carotenoids and podophyllotoxin analogues are now used in cancer chemotherapy $[36,37]$. There is accumulating evidence showing that crocetin, a carotenoid isolated from the plant saffron, exerts anti-tumor activity in certain types of cancers [5]. However, the role of crocetin in GC progression remains largely unclear. Therefore, it was imperative to explore the therapeutic effect of crocetin on GC and the underlying mechanisms.

Unrestrained cell proliferation, cell death resistance, angiogenesis, invasion and metastasis are well known as cancer hallmarks [11]. Thus, we first assayed the effects of crocetin on angiogenesis as what Umigai et al. had done using HUVECs [38] and we came to the same results presenting that crocetin inhibited angiogenesis by suppressing tube formation, proliferation and migration of HUVECs. Next, we examined the role of crocetin during GC progression. According to our results, cell proliferation of GC cells was obviously inhibited by crocetin in a dose-dependent manner. Similarly, GC growth in nude mice was also significantly inhibited due to subcutaneous injection of crocetin, which was consistent with investigation of Bathaie et al. and He et al. both showing the anti-proliferative activity of crocetin against GC $[39,40]$. Additionally, we observed that VM and mosaic vessels formation of GC cells were also significantly suppressed by crocetin treatment. Furthermore, the migration and invasion activity of crocetin-treated GC cells were reduced, and the cytoskeleton of GC cells shrank in the presentation of crocetin, suggesting that crocetin may effectively inhibit metastatic processes involved in GC.

Coincidently, an earlier study about the antitumor effects of crocetin in prostate cancer exhibited a similar conclusion [41].

Since attenuation of E-cadherin expression is generally accepted as a hallmark of the EMT process [42]. Thus, we detected the expression of EMT biomarkers to determine whether crocetin inhibits GC motility 
by reverting EMT in a manner similar to that found in the prostate cancer cell models mentioned above [41]. As expected, crocetin treatment leaded to a significant increase in expression of E-cadherin and on the contrary, expression of mesenchymal markers $\beta$-catenin and vimentin were reduced upon crocetin treatment. Multiple signaling pathways are known to be associated with EMT-mediated metastatic potential of malignant tumors, including Shh/Gli signaling, which has been proved to be involved in various stages of carcinogenesis in a variety of tumors [43]. A preliminary study done by Rangarajan et al. showed that the Shh/Gli pathway is significantly affected following treatment with purified crocetinic acid in pancreatic cancer stem cells [44]. Hence, we evaluated the effect of crocetin on Shh/Gli pathway. According to our results, crocetin treatment not only inhibited activation of Shh/Gli signaling, but also attenuated Hs-746-induced migration of HUVECs and HUVECs-induced Hs-746T cell migration through reducing secretion of Shh. Moreover, administration of recombinant Shh to crocetin-treated GC cells and HUVECs recovered EMT and antagonized the inhibitory effects of crocetin on cell proliferation, migration, angiogenesis and VM formation. These findings demonstrated that crocetin suppressed GC progression by inhibiting Shh/Gli signaling.

A number of previous researches have elucidated that crocetin can inhibit cancer with different mechanisms, such as anti-proliferative and anti-metastatic actions, as well as inducing apoptosis, inhibiting nucleic acid synthesis and enhancing anti-oxidative system [45]. In the present study, the data revealed that crocetin suppressed GC progression via inhibiting angiogenesis, cell proliferation and EMTmediated metastasis of GC cells and these inhibitory effects of crocetin were associated with attenuated activation of Shh/Gli signaling pathway. However, the related molecular targets involved in this process remain to be further explored and more in vivo studies are required to investigate the exact antitumor mechanisms of crocetin, providing experimental basis for the clinical application of crocetin in the treatment of GC.

\section{Conclusions}

Collectively, our findings demonstrated an important role of crocetin in suppressing GC progression via the inhibition of cell proliferation, migration and angiogenesis. In addition, these inhibitory effects of crocetin on GC progression were realized by inhibiting the activation of Shh signaling pathway. This study unveils the potential usefulness of crocetin to target GC therapy.

\section{Abbreviations}

ELISA: Enzyme linked immunosorbent assay; EMT:Epithelial mesenchymal transition; GC:Gastric cancer; HUVEC:Human Umbilical Vein Endothelial Cells; IHC:Immunohistochemistry; RT-qPCR:Quantitative Realtime PCR; Shh:Sonic hedgehog; VM:Vasculogenic mimicry

\section{Declarations}

\section{Ethics approval and consent to participate}


An institutional review board from the local Human Research Ethics Committee of Shanghai Cancer Center, Fudan University School of Medicine, China approved the study. All human participants provided informed consent. All animal experiments were also approved by the local Laboratory Animal Ethics Committee of Shanghai Cancer Center and conducted based on the Guide for the Care and Use Laboratory Animals of Shanghai Cancer Center. All samples tested for GC were anonymous in accordance with legal and ethical standards.

\section{Consent for publication}

All contributing authors agree to the publication of this article.

\section{Availability of data and materials}

All data are fully available without restrictions.

\section{Competing interests}

The authors declare that they have no competing interests.

\section{Funding}

This study was supported by grants from the National Natural Science foundation of China No. 81902403.

\section{Authors' contributions}

$\mathrm{YZ}$ and $\mathrm{YW}$ conceived the study design. $\mathrm{MZ}$ and $\mathrm{JH}$ performed experiments and collected all data. $\mathrm{MZ}$, JW and $\mathrm{YH}$ collected and analyzed data and drafted the manuscript. XD, JW and YH assisted in drafting and revising the manuscript. All authors read and approved the final manuscript.

\section{Acknowledgements}

We thank Pro. Dazhi Xu for his suggestion on this study.

\section{References}

1. Bray F, Ferlay J, Soerjomataram I, Siegel RL, Torre LA, Jemal A. Global cancer statistics 2018 : GLOBOCAN estimates of incidence and mortality worldwide for 36 cancers in 185 countries. CA Cancer J Clin. 2018;68:394-424.

2. Van Cutsem E, Sagaert X, Topal B, Haustermans K, Prenen H. Gastric cancer. Lancet. 2016;388:2654-64.

3. Patel TH, Cecchini M. Targeted Therapies in Advanced Gastric Cancer. Curr Treat Options Oncol. 2020;21:70. 
4. Riquelme I, Letelier P, Riffo-Campos AL, Brebi P, Roa JC. Emerging Role of miRNAs in the Drug Resistance of Gastric Cancer. Int J Mol Sci. 2016;17:424.

5. Colapietro A, Mancini A, D'Alessandro AM, Festuccia C. Crocetin and Crocin from Saffron in Cancer Chemotherapy and Chemoprevention. Anticancer Agents Med Chem. 2019;19:38-47.

6. Hashemi M, Hosseinzadeh $\mathrm{H}$. A comprehensive review on biological activities and toxicology of crocetin. Food Chem Toxicol. 2019;130:44-60.

7. Chen B, Hou ZH, Dong Z, Li CD. Crocetin downregulates the proinflammatory cytokines in methylcholanthrene-induced rodent tumor model and inhibits COX-2 expression in cervical cancer cells. Biomed Res Int. 2015;2015:829513.

8. Nam KN, Park YM, Jung HJ, Lee JY, Min BD, Park SU, Jung WS, Cho KH, Park JH, Kang I, et al. Antiinflammatory effects of crocin and crocetin in rat brain microglial cells. Eur $\mathrm{J}$ Pharmacol. 2010;648:110-6.

9. Zhuang X, Dong A, Wang R, Shi A. Crocetin treatment inhibits proliferation of colon cancer cells through down-regulation of genes involved in the inflammation. Saudi J Biol Sci. 2018;25:1767-71.

10. Yamashita S-I, Kakinuma T, Umigai N, Takara T, et al. Safety evaluation of excessive intake of crocetin in healthy adult volunteers: - A randomized, double-blind, placebo-controlled, parallel-group comparison trial. Japanese Pharmacology Therapeutics. 2018;46:393-401.

11. Hanahan D, Weinberg RA. The hallmarks of cancer. Cell. 2000;100:57-70.

12. Zetter BR. Angiogenesis and tumor metastasis. Annu Rev Med. 1998;49:407-24.

13. Potente $M$, Gerhardt $H$, Carmeliet P. Basic and therapeutic aspects of angiogenesis. Cell. 2011;146:873-87.

14. Ricci-Vitiani L, Pallini R, Biffoni M, et al. Tumour vascularization via endothelial differentiation of glioblastoma stem-like cells. Nature. 2010;468:824-8.

15. Fernández-Cortés M, Delgado-Bellido D, Oliver FJ. Vasculogenic Mimicry: Become an Endothelial Cell "But Not So Much". Front Oncol. 2019;9:803.

16. Maniotis AJ, Folberg R, Hess A, et al. Vascular channel formation by human melanoma cells in vivo and in vitro: vasculogenic mimicry. Am J Pathol. 1999;155:739-52.

17. Schnegg $\mathrm{Cl}$, Yang MH, Ghosh SK, Hsu MY. Induction of Vasculogenic Mimicry Overrides VEGF-A Silencing and Enriches Stem-like Cancer Cells in Melanoma. Cancer Res. 2015;75:1682-90.

18. Meng J, Chen S, Lei YY, et al. Hsp90ß promotes aggressive vasculogenic mimicry via epithelialmesenchymal transition in hepatocellular carcinoma. Oncogene. 2019;38:228-43.

19. Camorani S, Crescenzi E, Gramanzini M, Fedele M, Zannetti A, Cerchia L. Aptamer-mediated impairment of EGFR-integrin av $\beta 3$ complex inhibits vasculogenic mimicry and growth of triplenegative breast cancers. Sci Rep. 2017;7:46659.

20. Wang $\mathrm{H}$, Huang B, Li BM, et al. ZEB1-mediated vasculogenic mimicry formation associates with epithelial-mesenchymal transition and cancer stem cell phenotypes in prostate cancer. J Cell Mol Med 2018. 
21. Zhao B, Wu M, Hu Z, et al. Thrombin is a therapeutic target for non-small-cell lung cancer to inhibit vasculogenic mimicry formation. Signal Transduct Target Ther. 2020;5:117.

22. Li W, Zong S, Shi Q, Li H, Xu J, Hou F. Hypoxia-induced vasculogenic mimicry formation in human colorectal cancer cells: Involvement of HIF-1a, Claudin-4, and E-cadherin and Vimentin. Sci Rep. 2016;6:37534.

23. Zang $M$, Zhang $Y$, Zhang B, et al. CEACAM6 promotes tumor angiogenesis and vasculogenic mimicry in gastric cancer via FAK signaling. Biochim Biophys Acta. 2015;1852:1020-8.

24. Cao Z, Bao M, Miele L, Sarkar FH, Wang Z, Zhou Q. Tumour vasculogenic mimicry is associated with poor prognosis of human cancer patients: a systemic review and meta-analysis. Eur $\mathrm{J}$ Cancer. 2013;49(18):3914-23.

25. Ge H, Luo H. Overview of advances in vasculogenic mimicry - a potential target for tumor therapy. Cancer Manag Res. 2018;10:2429-37.

26. Luo Q, Wang J, Zhao W, et al. Vasculogenic mimicry in carcinogenesis and clinical applications. J Hematol Oncol. 2020;13:19.

27. Di Mauro C, Rosa R, D'Amato V, et al. Hedgehog signalling pathway orchestrates angiogenesis in triple-negative breast cancers. Br J Cancer. 2017;116:1425-35.

28. Salaritabar A, Berindan-Neagoe I, Darvish B, et al. Targeting Hedgehog signaling pathway: Paving the road for cancer therapy. Pharmacol Res. 2019;141:466-80.

29. Dutzmann J, Koch A, Weisheit S, et al. Sonic hedgehog-dependent activation of adventitial fibroblasts promotes neointima formation. Cardiovasc Res. 2017;113:1653-63.

30. Carpenter RL, Ray H. Safety and Tolerability of Sonic Hedgehog Pathway Inhibitors in Cancer. Drug Saf. 2019;42:263-79.

31. Ferro V, Liu L, Johnstone KD, et al. Discovery of PG545: a highly potent and simultaneous inhibitor of angiogenesis, tumor growth, and metastasis. J Med Chem. 2012;55:3804-13.

32. Zang MD, Hu L, Fan ZY, et al. Luteolin suppresses gastric cancer progression by reversing epithelialmesenchymal transition via suppression of the Notch signaling pathway. J Transl Med. 2017;15:52.

33. Wang SM, Tie J, Wang WL, et al. POU2F2-oriented network promotes human gastric cancer metastasis. Gut. 2016;65:1427-38.

34. Ham IH, Oh HJ, Jin H, et al. Targeting interleukin- 6 as a strategy to overcome stroma-induced resistance to chemotherapy in gastric cancer. Mol Cancer. 2019;18:68.

35. Housman G, Byler S, Heerboth S, et al. Drug resistance in cancer: an overview. Cancers (Basel). 2014;6:1769-92.

36. Saklani A, Kutty SK. Plant-derived compounds in clinical trials. Drug Discov Today. 2008;13:161-71.

37. Siddikuzzaman GC, Berlin Grace VM. All trans retinoic acid and cancer. Immunopharmacol Immunotoxicol. 2011;33:241-9.

38. Umigai N, Tanaka J, Tsuruma K, Shimazawa M, Hara H. Crocetin, a carotenoid derivative, inhibits VEGF-induced angiogenesis via suppression of p38 phosphorylation. Curr Neurovasc Res. 
2012;9:102-9.

39. Bathaie SZ, Hoshyar R, Miri H, Sadeghizadeh M. Anticancer effects of crocetin in both human adenocarcinoma gastric cancer cells and rat model of gastric cancer. Biochem Cell Biol. 2013;91:397-403.

40. He K, Si P, Wang H, et al. Crocetin induces apoptosis of BGC-823 human gastric cancer cells. Mol Med Rep. 2014;9:521-6.

41. Festuccia C, Mancini A, Gravina GL, et al. Antitumor effects of saffron-derived carotenoids in prostate cancer cell models. Biomed Res Int. 2014;2014:135048.

42. Hur K, Toiyama Y, Takahashi M, et al. MicroRNA-200c modulates epithelial-to-mesenchymal transition (EMT) in human colorectal cancer metastasis. Gut. 2013;62:1315-26.

43. Niyaz M, Khan MS, Mudassar S. Hedgehog Signaling: An Achilles' Heel in Cancer. Transl Oncol. 2019;12:1334-44.

44. Rangarajan $P$, Subramaniam D, Paul S, et al. Crocetinic acid inhibits hedgehog signaling to inhibit pancreatic cancer stem cells. Oncotarget. 2015;6:27661-73.

45. Moradzadeh M, Sadeghnia HR, Tabarraei A, Sahebkar A. Anti-tumor effects of crocetin and related molecular targets. J Cell Physiol. 2018;233:2170-82.

\section{Figures}

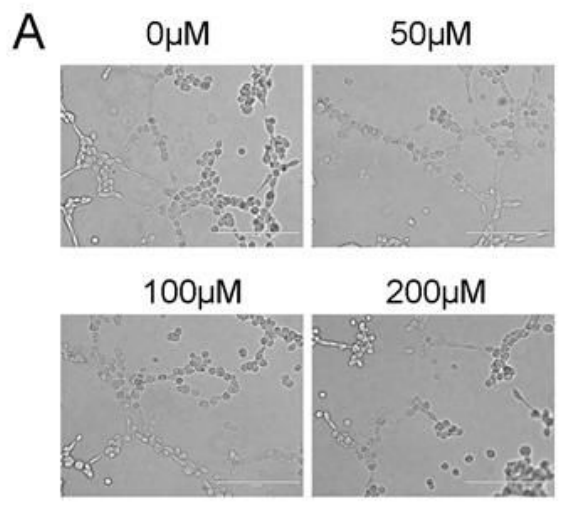

$\mathrm{E}$

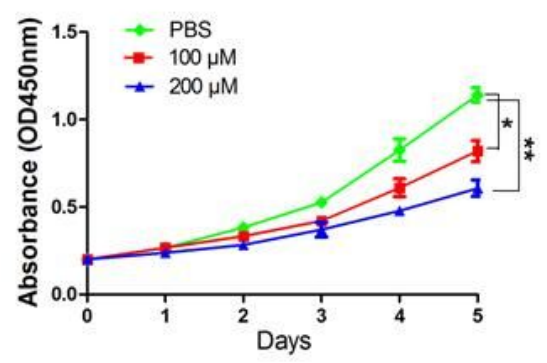

B

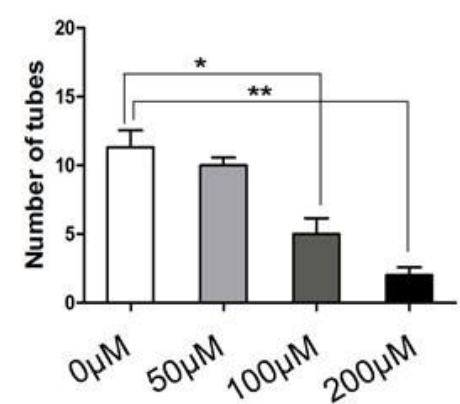

OHM

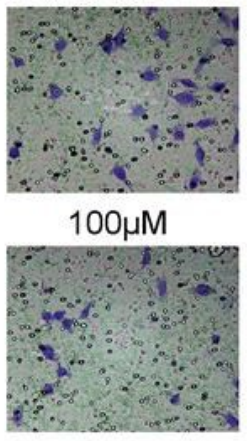

C

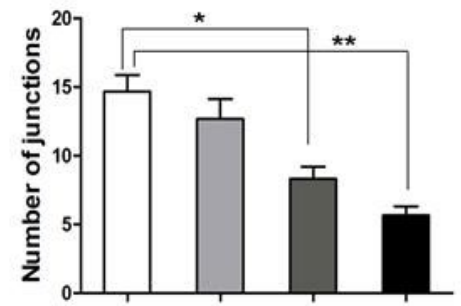

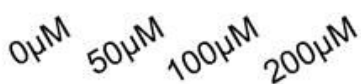

F

HUVEC

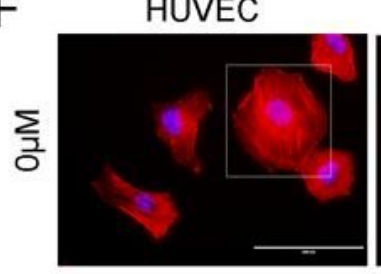

F-actin

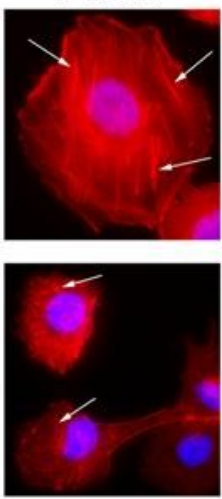

Figure 1 
Crocetin inhibits tube formation, cell proliferation, and migration of HUVEC cells. a. Crocetin inhibits tube formation of HUVECs with various concentration. b-c. Bar charts show the number of tubes (b) and number of intersections (c) in different groups with or without crocetin. $d$. The proliferation of HUVECs is inhibited in crocetin treatment groups compared with the control groups. e. Crocetin inhibits cell migration of HUVECs. f. F-actin staining shows that cell cytoskeleton was broken by crocetin. Data are represented as mean \pm SEM of three independent experiments. Student's t-test, $* \mathrm{P}<0.05, * * \mathrm{P}<0.01$.
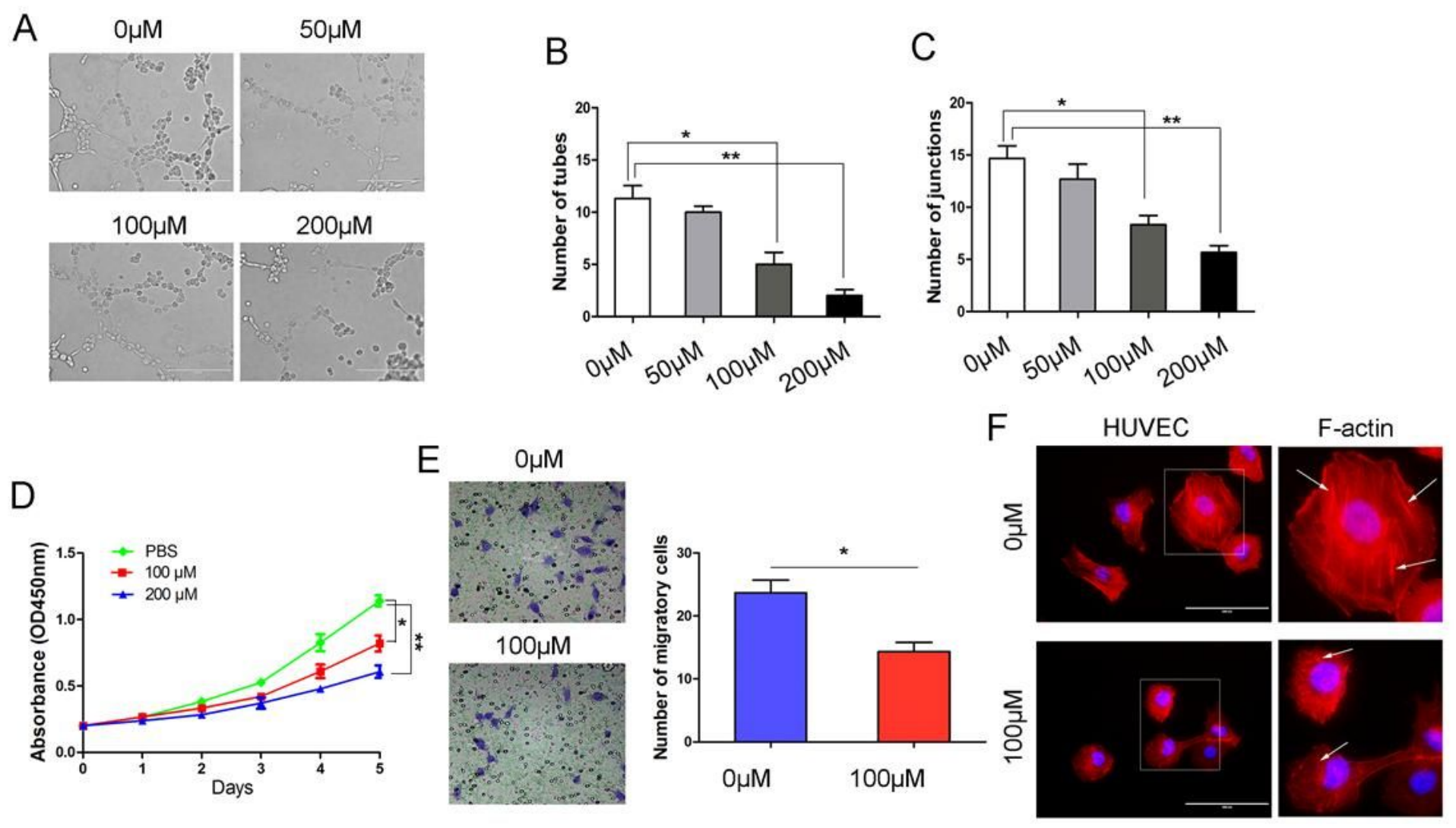

\section{Figure 1}

Crocetin inhibits tube formation, cell proliferation, and migration of HUVEC cells. a. Crocetin inhibits tube formation of HUVECs with various concentration. b-c. Bar charts show the number of tubes (b) and number of intersections (c) in different groups with or without crocetin. $d$. The proliferation of HUVECs is inhibited in crocetin treatment groups compared with the control groups. e. Crocetin inhibits cell migration of HUVECs. f. F-actin staining shows that cell cytoskeleton was broken by crocetin. Data are represented as mean \pm SEM of three independent experiments. Student's t-test, ${ }^{*} P<0.05,{ }^{\star} * P<0.01$. 
A

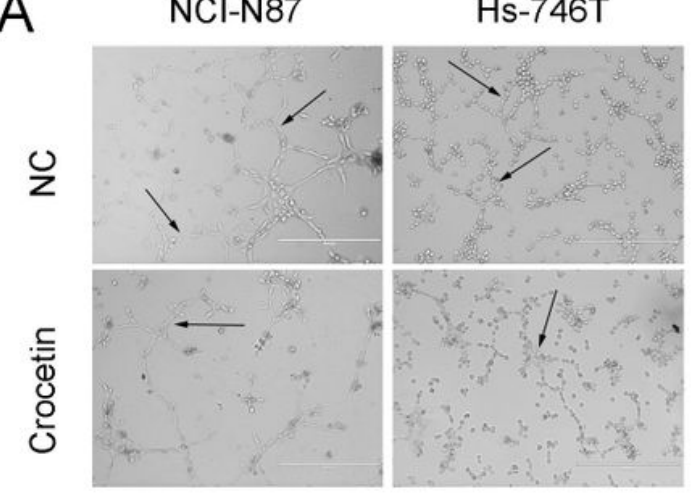

D

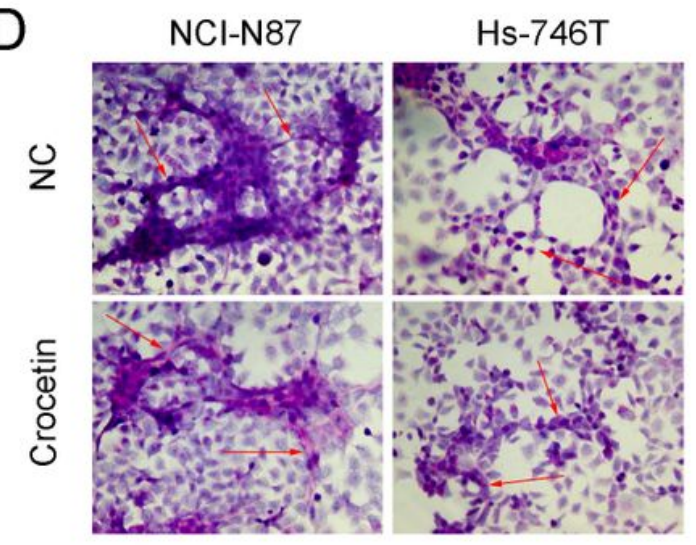

G
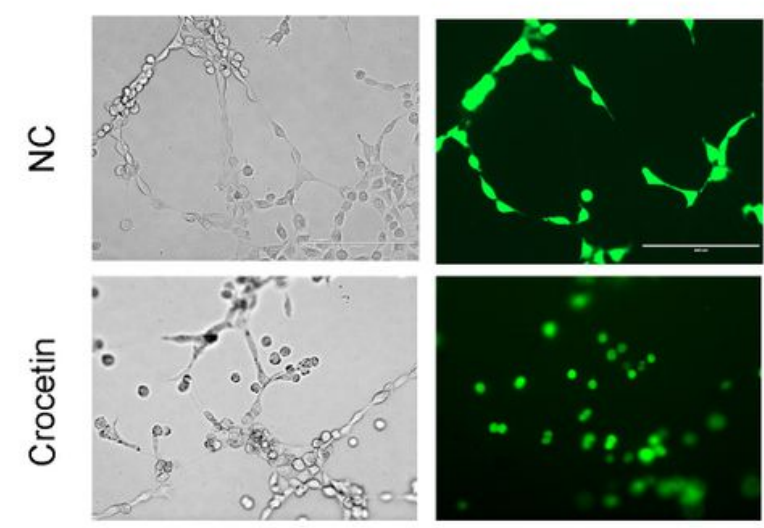

B

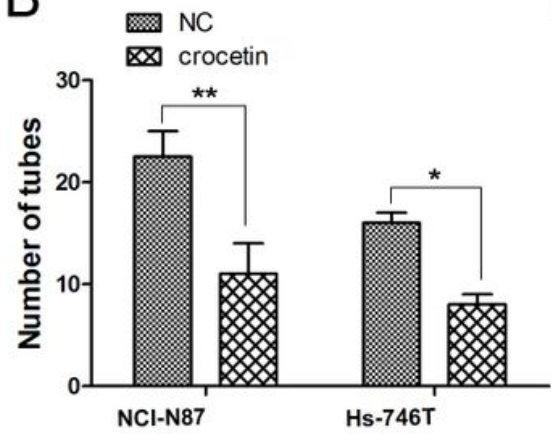

$E$

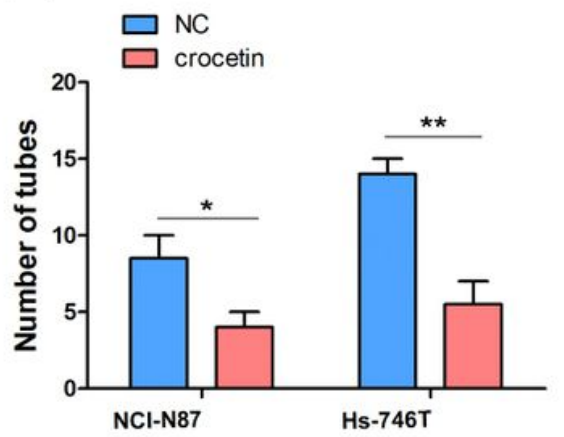

C

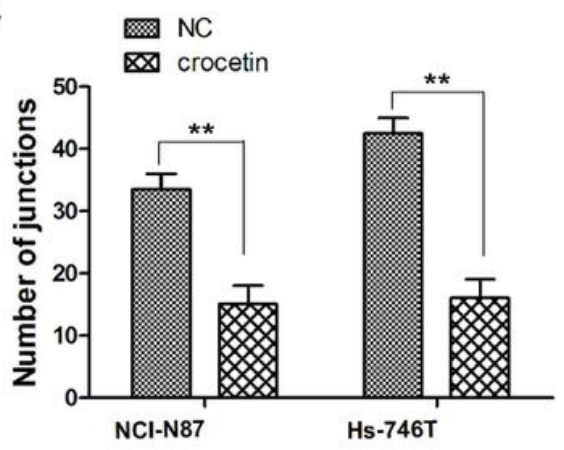

$\mathrm{F}$

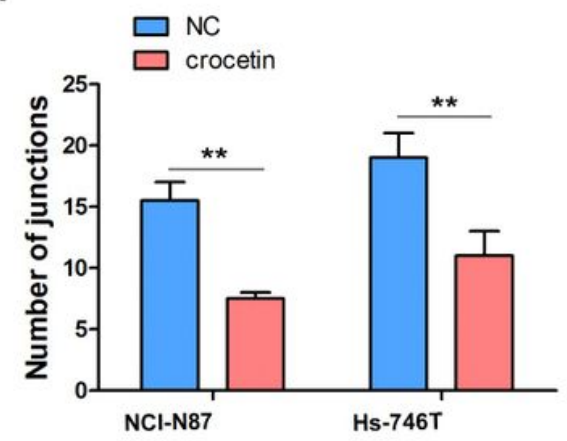

Merge

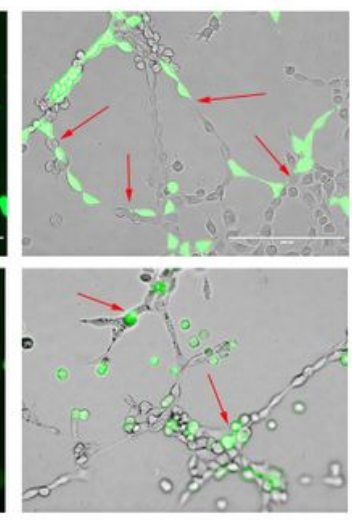

$\mathrm{H}$

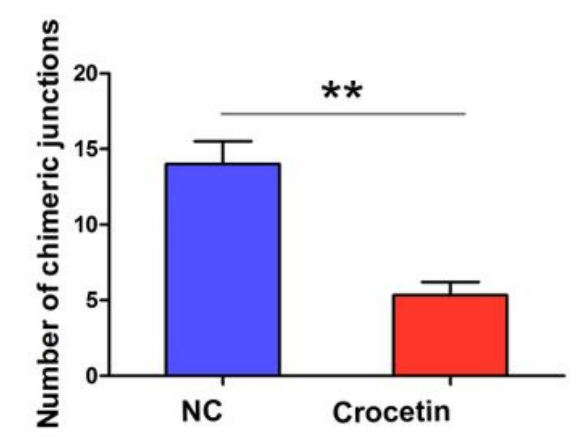

Figure 2

Crocetin inhibits VM tubes and mosaic vessels formation in GC cells. a. Less vessel-like structures defined as VM are observed in NCI-N87 and Hs-746T GC cells with $100 \mu \mathrm{M}$ crocetin treatment compared with control groups. b-c. Bar charts show the number of VM tubes (b) and number of VM intersections (c) in different groups. d. 3D-culture shows effect of crocetin on VM structures by PAS staining. e-f. The number of VM tubes (e) and number of VM intersections (f) in different groups. $g$. The mixed cells are cultured on Matrigel according to 1:1 with HUVECs without EGFP label and Hs-746T GC cells which have EGFP label. Mosaic vessels are destroyed in crocetin treatment groups. $\mathrm{H}$. The number of mosaic vessel intersections in groups with or without crocetin. Data are represented as mean \pm SEM of three independent experiments. Student's t-test, ${ }^{*} \mathrm{P}<0.05, * * \mathrm{P}<0.01$. 


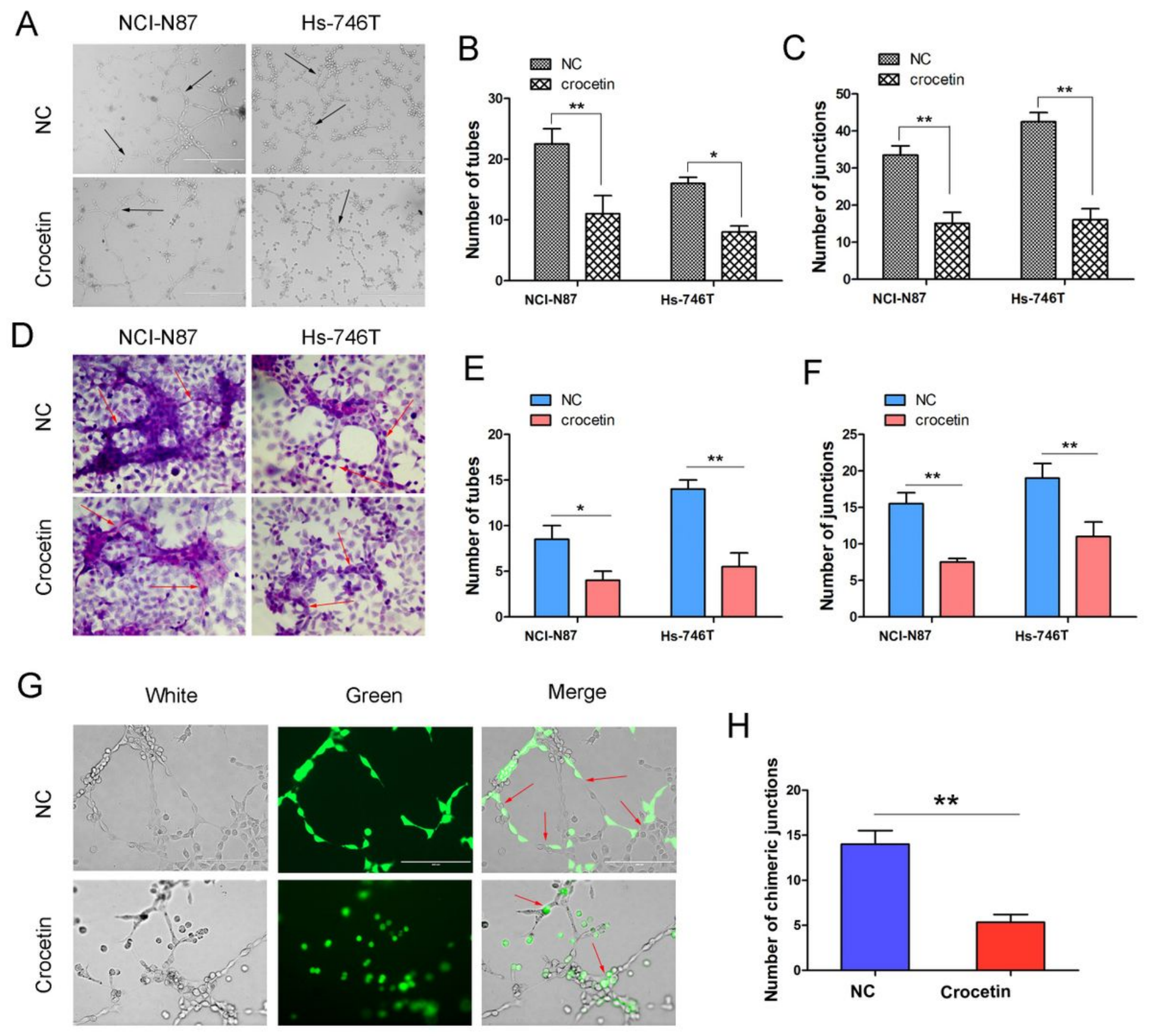

Figure 2

Crocetin inhibits VM tubes and mosaic vessels formation in GC cells. a. Less vessel-like structures defined as VM are observed in NCI-N87 and Hs-746T GC cells with $100 \mu \mathrm{M}$ crocetin treatment compared with control groups. b-c. Bar charts show the number of VM tubes (b) and number of VM intersections (c) in different groups. d. 3D-culture shows effect of crocetin on VM structures by PAS staining. e-f. The number of VM tubes (e) and number of VM intersections (f) in different groups. g. The mixed cells are cultured on Matrigel according to 1:1 with HUVECs without EGFP label and Hs-746T GC cells which have EGFP label. Mosaic vessels are destroyed in crocetin treatment groups. $\mathrm{H}$. The number of mosaic vessel intersections in groups with or without crocetin. Data are represented as mean \pm SEM of three independent experiments. Student's t-test, ${ }^{*} \mathrm{P}<0.05,{ }^{*} \mathrm{P}<0.01$. 


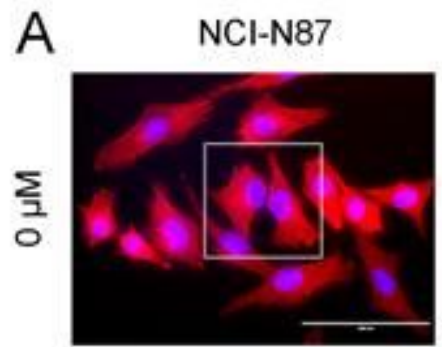

F-actin

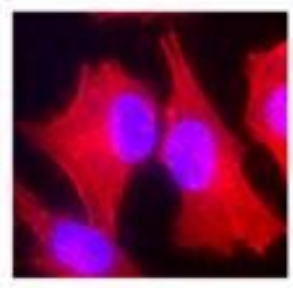

B

Hs-746T

F-actin
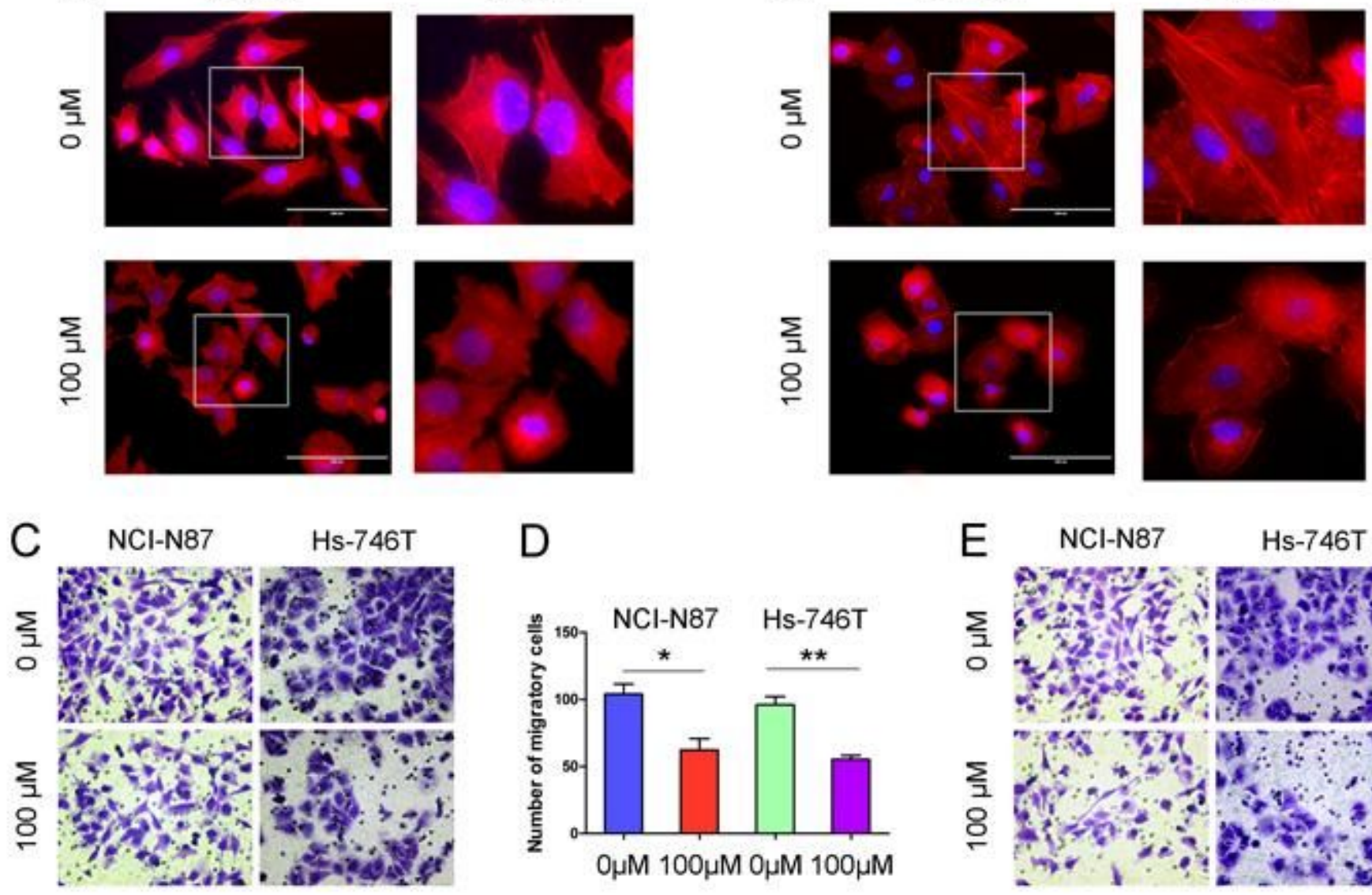

D

E $\quad \mathrm{NCl}-\mathrm{N} 87$

Hs-746T
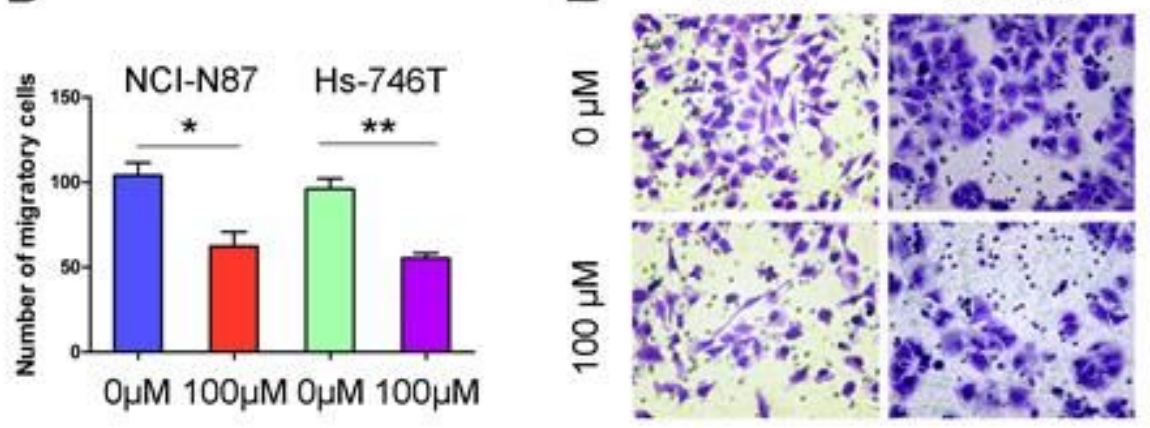

F

\section{G}

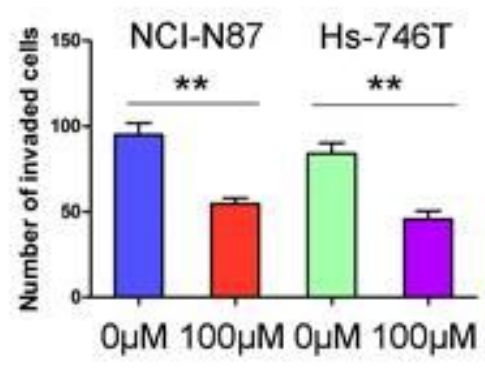

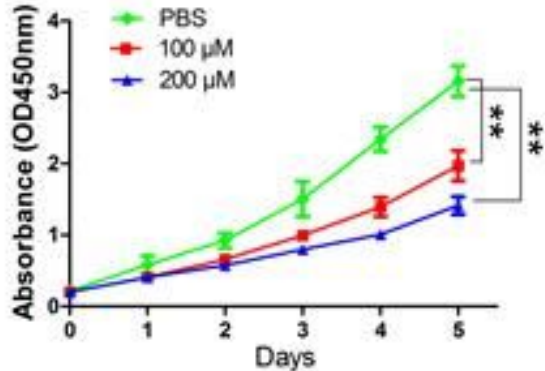

$\mathrm{NCl}-\mathrm{N} 87$
$\mathrm{H}$

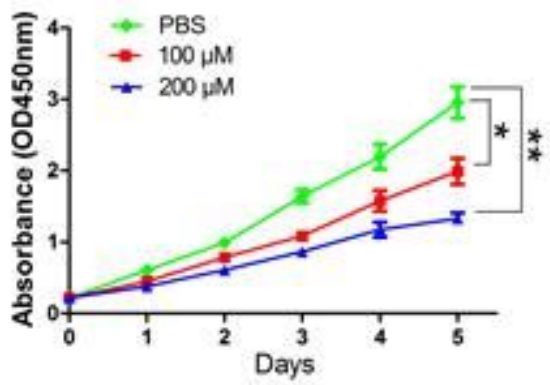

Hs-746T

\section{Figure 3}

Crocetin inhibits cell migration and proliferation in GC cells. a-b. More shrinking NCI N87 (a) and Hs-746T (b) GC cells are found in crocetin treatment groups compared with control groups. c. Effects of crocetin on migration of GC cells. $d$. Number of cell migration with or without crocetin. e. Effects of crocetin on invasion of GC cells. f. Number of cell invasion with or without crocetin. g-h. Crocetin treatment suppresses cell proliferation in NCI N87 (g) and Hs-746T (h) GC cells. Data are represented as mean \pm SEM of three independent experiments. Student's t-test, * $P<0.05, * \star P<0.01$. 


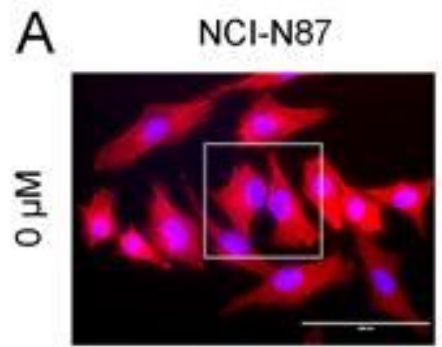

F-actin

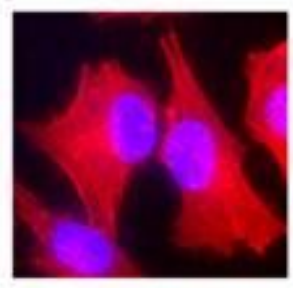

B

Hs-746T

F-actin
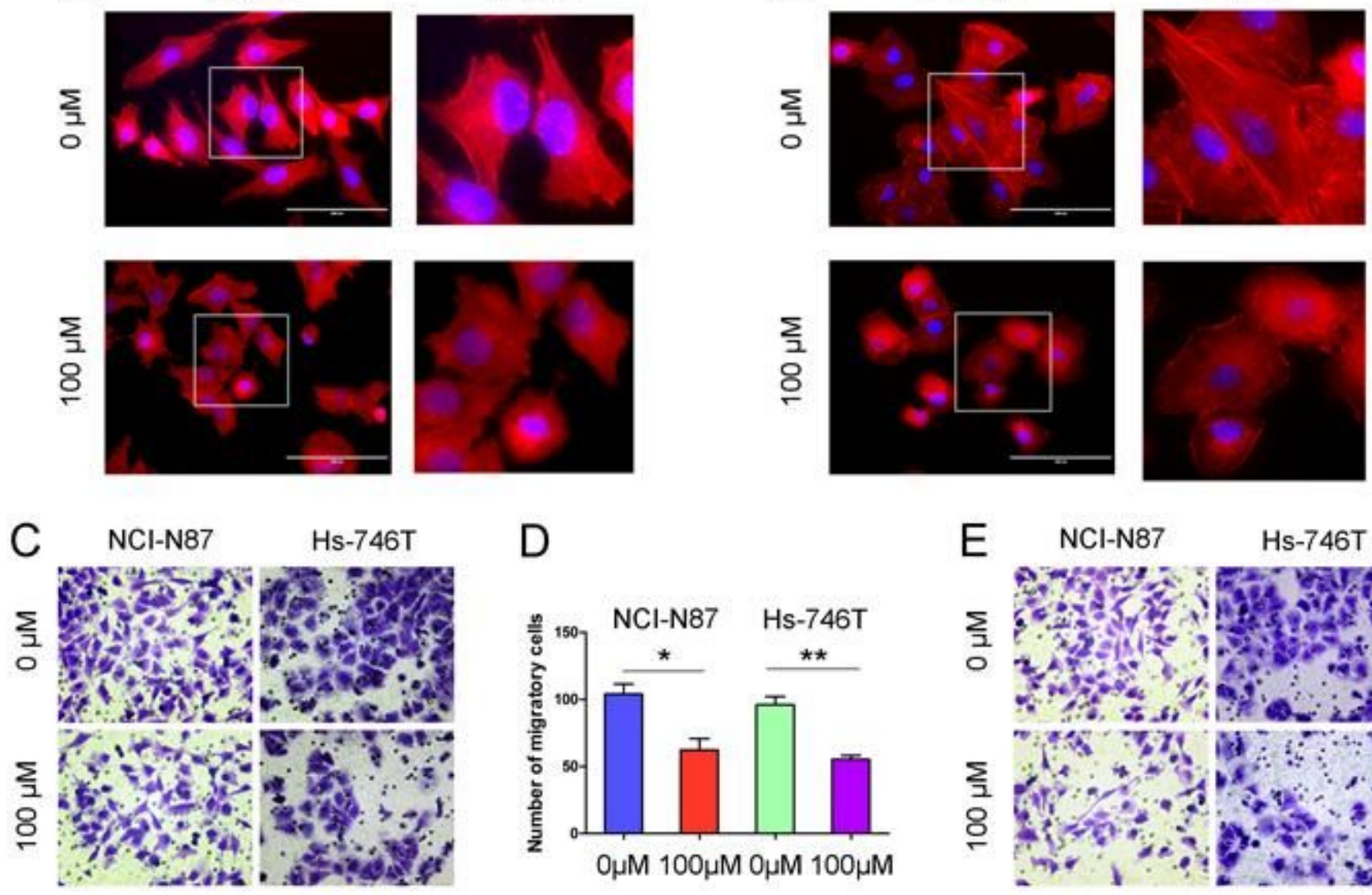

D

E $\quad \mathrm{NCl}-\mathrm{N} 87$

Hs-746T
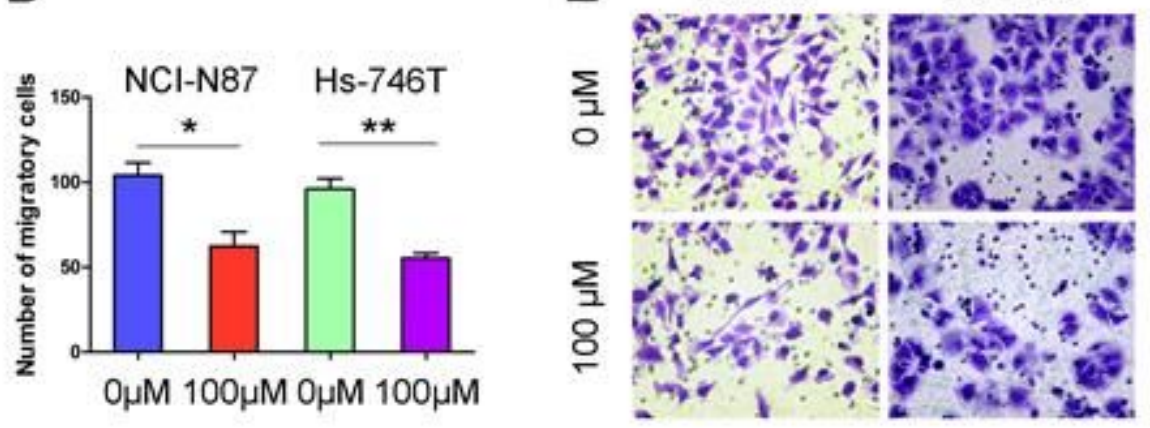

F

\section{G}

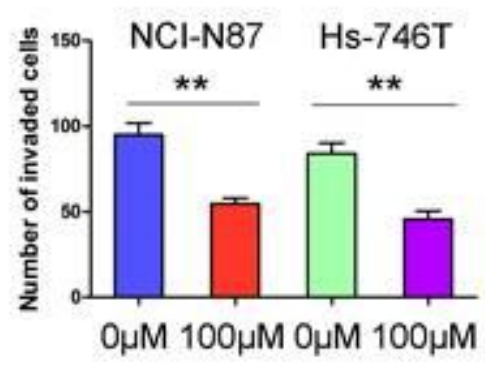

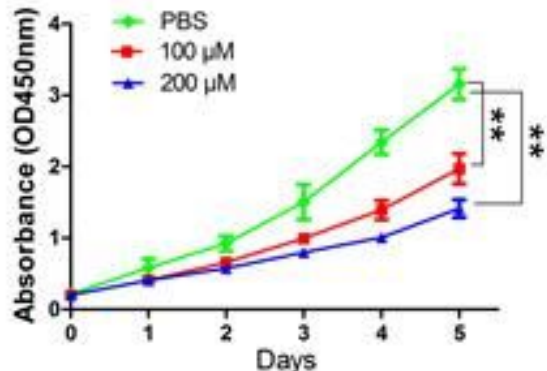

$\mathrm{NCl}-\mathrm{N} 87$
$\mathrm{H}$

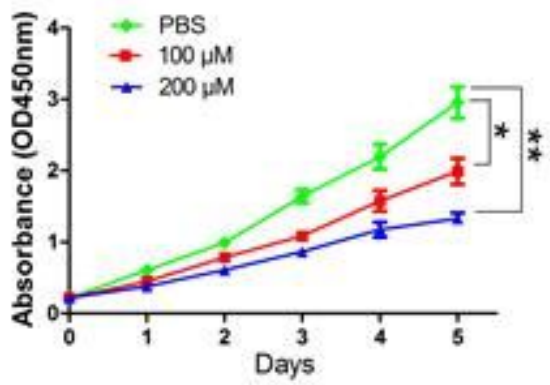

Hs-746T

\section{Figure 3}

Crocetin inhibits cell migration and proliferation in GC cells. a-b. More shrinking NCI N87 (a) and Hs-746T (b) GC cells are found in crocetin treatment groups compared with control groups. c. Effects of crocetin on migration of GC cells. $d$. Number of cell migration with or without crocetin. e. Effects of crocetin on invasion of GC cells. f. Number of cell invasion with or without crocetin. g-h. Crocetin treatment suppresses cell proliferation in NCI N87 (g) and Hs-746T (h) GC cells. Data are represented as mean \pm SEM of three independent experiments. Student's t-test, * $P<0.05, * \star P<0.01$. 
A

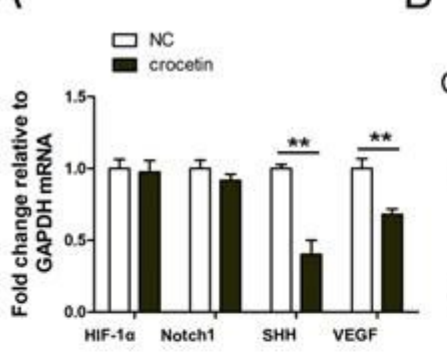

B

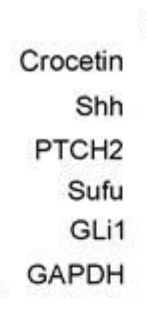

D

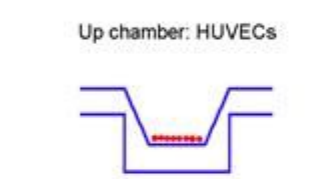

Down chamber: $\pm \mathrm{Hs}-746 \mathrm{~T} \pm$ Crocetin

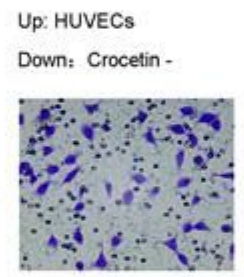

Up: HUVECs

Down: Crocetin +

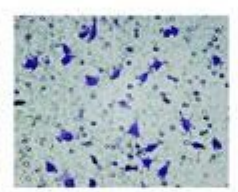

Up: HUVECs

Down: Hs-746T

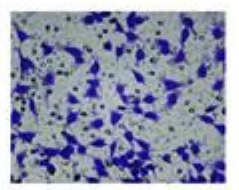

Up: HUVECs

Down: Hs-746T+Crocetin

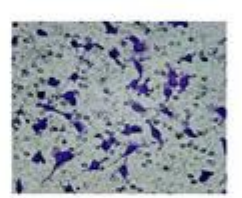

E

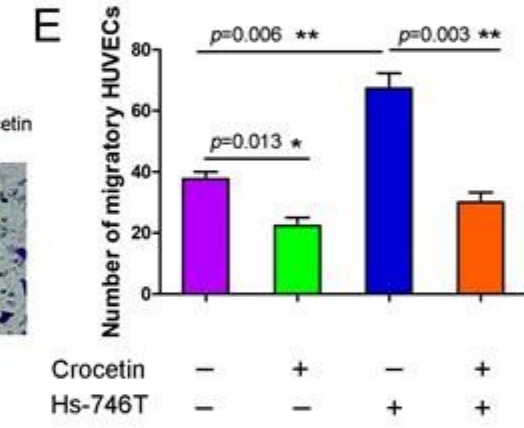

$\mathrm{F}$

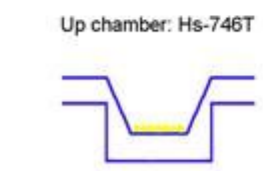

Down chamber: \pm HUVECs \pm Crocetin

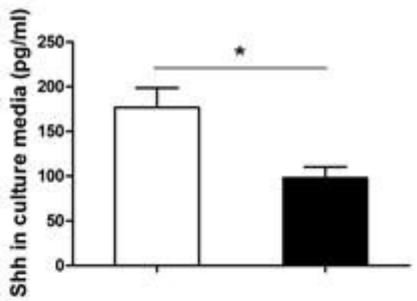

Crocetin - +
G

Down: HUVECs+Crocetin
Up: Hs-746T

Down: Crocetin +

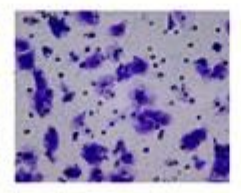

Up: Hs-746T

Down: HUVECs

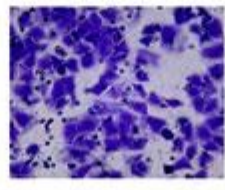

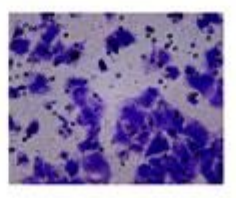

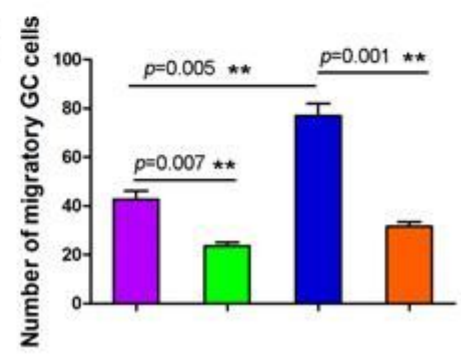

$\begin{array}{lllll}\text { Crocetin } & - & + & - & + \\ \text { HUVECS } & - & - & + & +\end{array}$

\section{Figure 4}

Crocetin inhibits cell migration through Shh signaling pathway. a. RT-PCR shows that Shh expression decreased significantly with crocetin treatment. b. Crocetin inhibits Shh signaling pathway on protein level. c. Effects of crocetin on Shh secretion in Hs-746T GC cells and HUVECs. d. Crocetin suppresses migration of HUVECs induced by Hs-746T. e. Number of migratory cells of HUVECs induced by Hs-746T with or without crocetin. f. Crocetin suppresses migration of Hs-746T GC cells induced by HUVECs. $g$. Number of migratory cells of Hs-746T induced by HUVECs with or without crocetin. Data are represented as mean \pm SEM of three independent experiments. Student's t-test, $* \mathrm{P}<0.05,{ }^{\star *} \mathrm{P}<0.01$. 
A

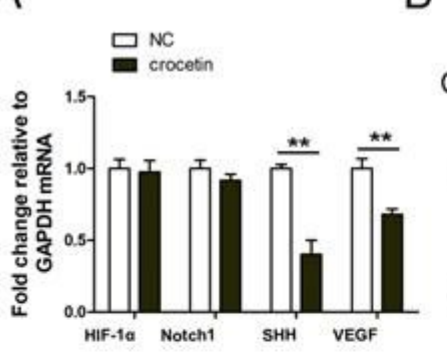

B

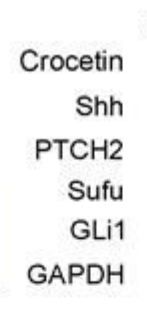

D

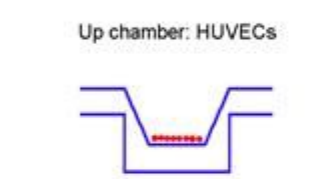

Down chamber: $\pm \mathrm{Hs}-746 \mathrm{~T} \pm$ Crocetin

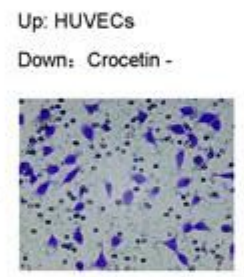

Up: HUVECs

Down: Crocetin +

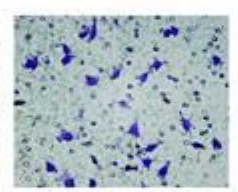

Up: HUVECs

Down: Hs-746T

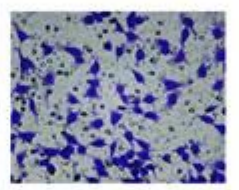

Up: HUVECs

Down: Hs-746T+Crocetin

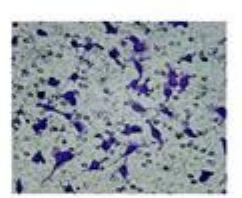

E

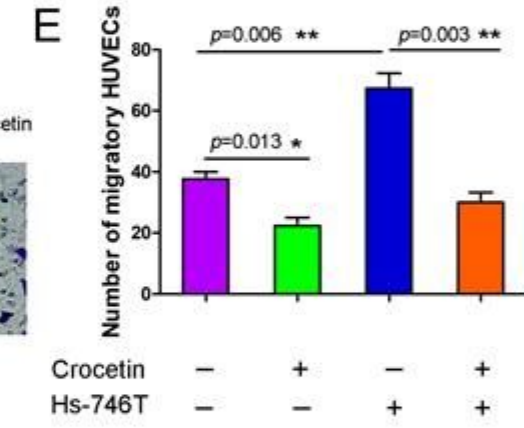

$\mathrm{F}$

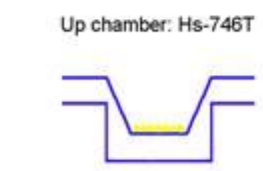

Down chamber: \pm HUVECs \pm Crocetin

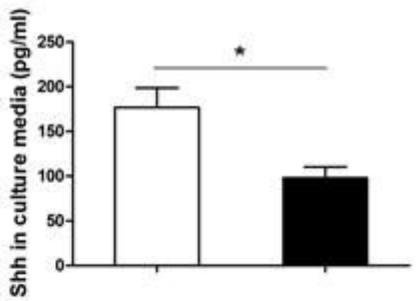

Crocetin - +
G

Down: HUVECs+Crocetin
Up: Hs-746T

Down: Crocetin +

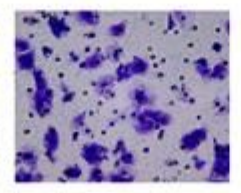

Up: Hs-746T

Down: HUVECs

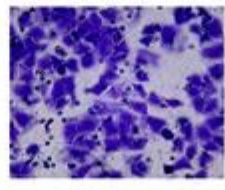

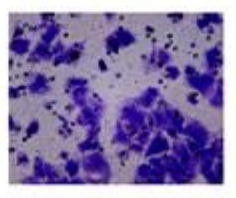

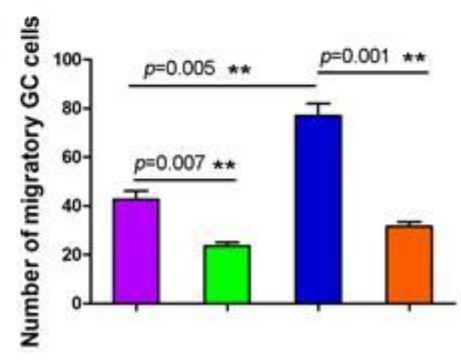

$\begin{array}{lllll}\text { Crocetin } & - & + & - & + \\ \text { HUVECS } & - & - & + & +\end{array}$

\section{Figure 4}

Crocetin inhibits cell migration through Shh signaling pathway. a. RT-PCR shows that Shh expression decreased significantly with crocetin treatment. b. Crocetin inhibits Shh signaling pathway on protein level. c. Effects of crocetin on Shh secretion in Hs-746T GC cells and HUVECs. d. Crocetin suppresses migration of HUVECs induced by Hs-746T. e. Number of migratory cells of HUVECs induced by Hs-746T with or without crocetin. f. Crocetin suppresses migration of Hs-746T GC cells induced by HUVECs. $g$. Number of migratory cells of Hs-746T induced by HUVECs with or without crocetin. Data are represented as mean \pm SEM of three independent experiments. Student's t-test, $* \mathrm{P}<0.05,{ }^{\star *} \mathrm{P}<0.01$. 
A

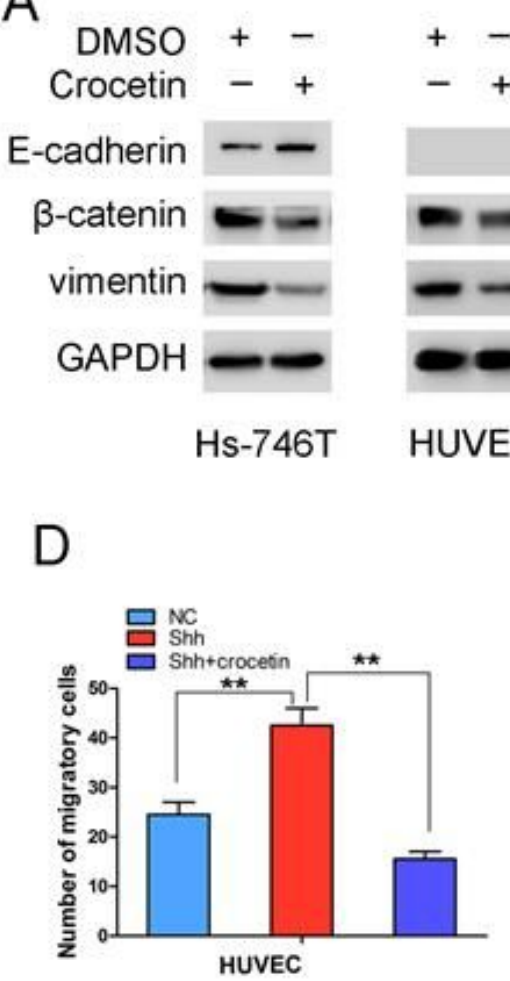

G

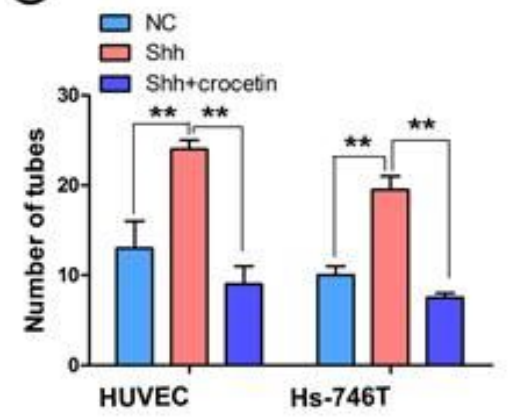

B

NC +--
Sh-GLi1 --+-
N-Shh -+++
Crocetin ---+

E-cadherin

$\beta$-catenin

vimentin

GAPDH

E

F
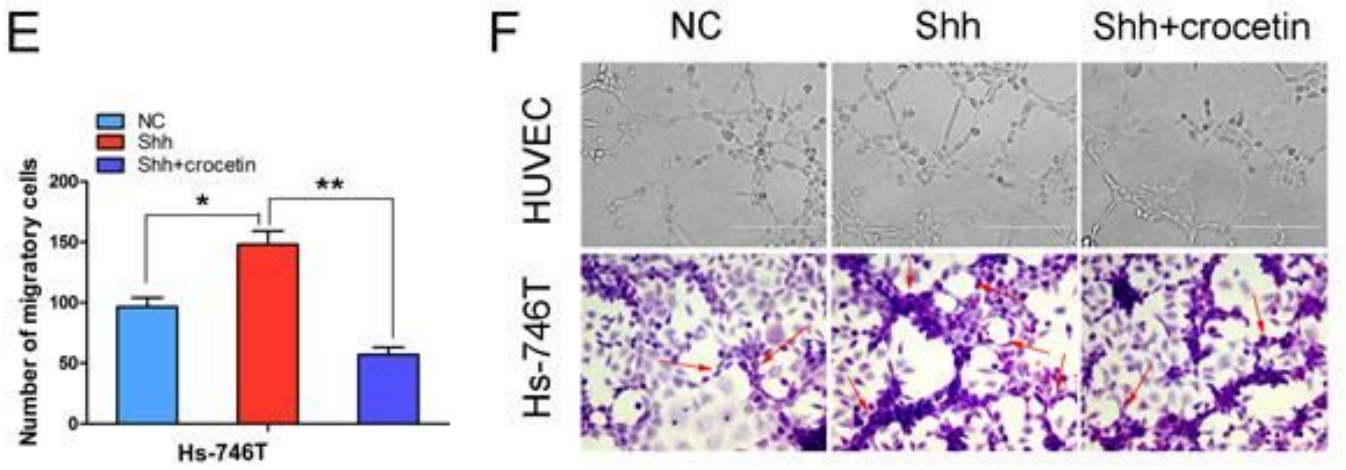

C

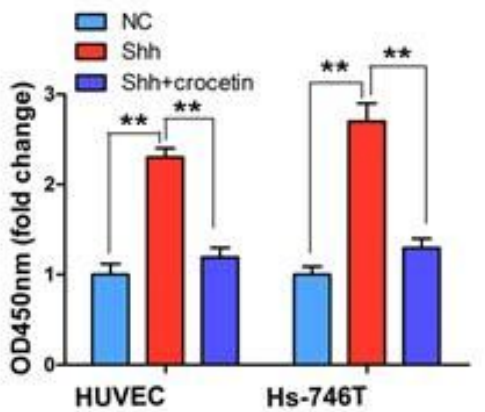

HUVEC Hs-746T

$\mathrm{H}$

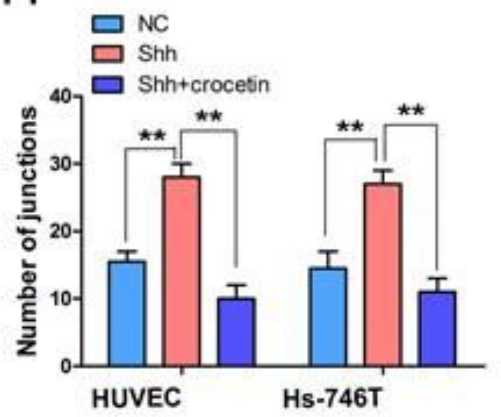

\section{Figure 5}

Recombinant Shh antagonizes the effects of crocetin on GC cells and HUVECs. a. Crocetin inhibits EMT in GC cells and HUVECs. b. Effects of crocetin and sh-Gli1 on EMT are antagonized by recombinant Shh. c-f. Effects of recombinant Shh on cell proliferation of HUVECs and Hs-746T (c), cell migration of HUVECs (d) and Hs-746T (e), HUVEC tube and VM formation (f) with or without crocetin. Number of tubes (g) and number of junctions $(\mathrm{h})$ with or without crocetin. Data are represented as mean \pm SEM of three independent experiments. Student's t-test, ${ }^{*} \mathrm{P}<0.05,{ }^{\star \star} \mathrm{P}<0.01$. 
A

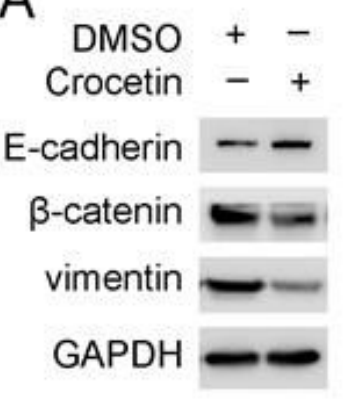

Hs-746T
$+-$

$-+$

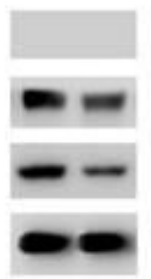

HUVEC
B

$\mathrm{NC}+---$
Sh-GLi1 --+-
N-Shh -+++
Crocetin --++

\section{E-cadherin}

$\beta$-catenin

vimentin

GAPDH

C

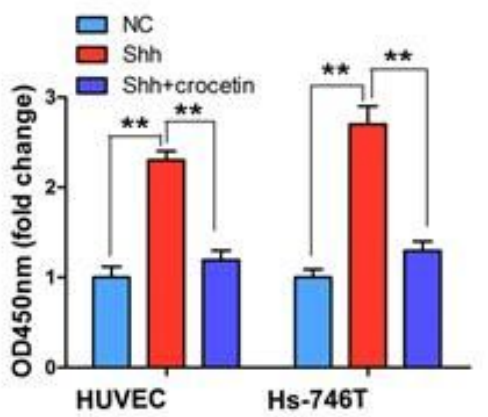

F

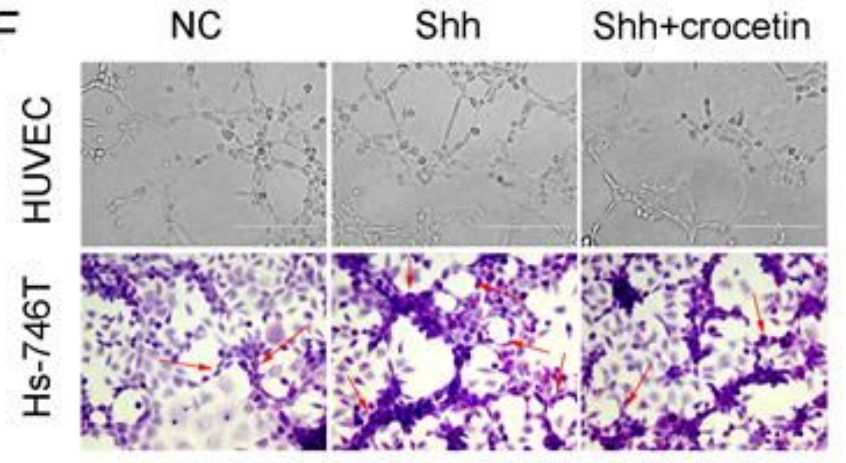

G

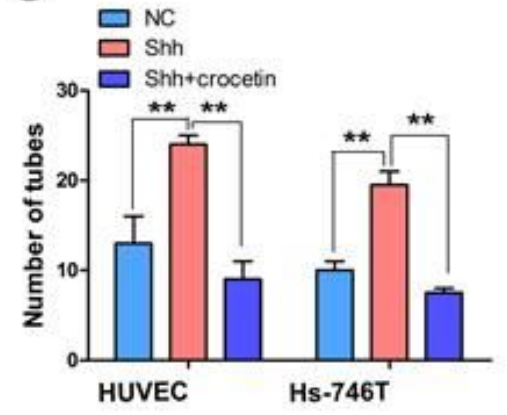

E

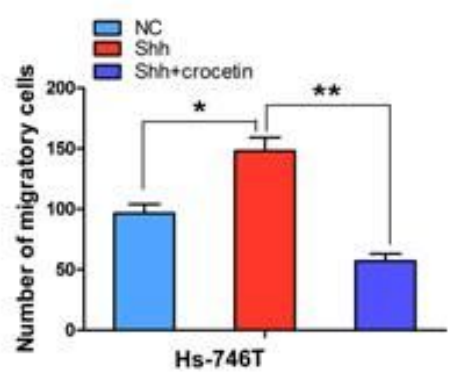

$\mathrm{H}$

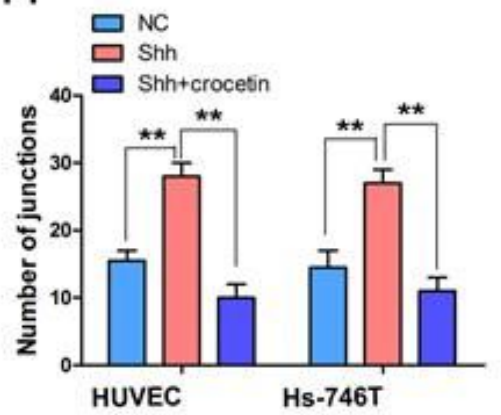

\section{Figure 5}

Recombinant Shh antagonizes the effects of crocetin on GC cells and HUVECs. a. Crocetin inhibits EMT in GC cells and HUVECs. b. Effects of crocetin and sh-Gli1 on EMT are antagonized by recombinant Shh. c-f. Effects of recombinant Shh on cell proliferation of HUVECs and Hs-746T (c), cell migration of HUVECs (d) and Hs-746T (e), HUVEC tube and VM formation (f) with or without crocetin. Number of tubes (g) and number of junctions (h) with or without crocetin. Data are represented as mean \pm SEM of three independent experiments. Student's t-test, ${ }^{*} \mathrm{P}<0.05, * * \mathrm{P}<0.01$. 

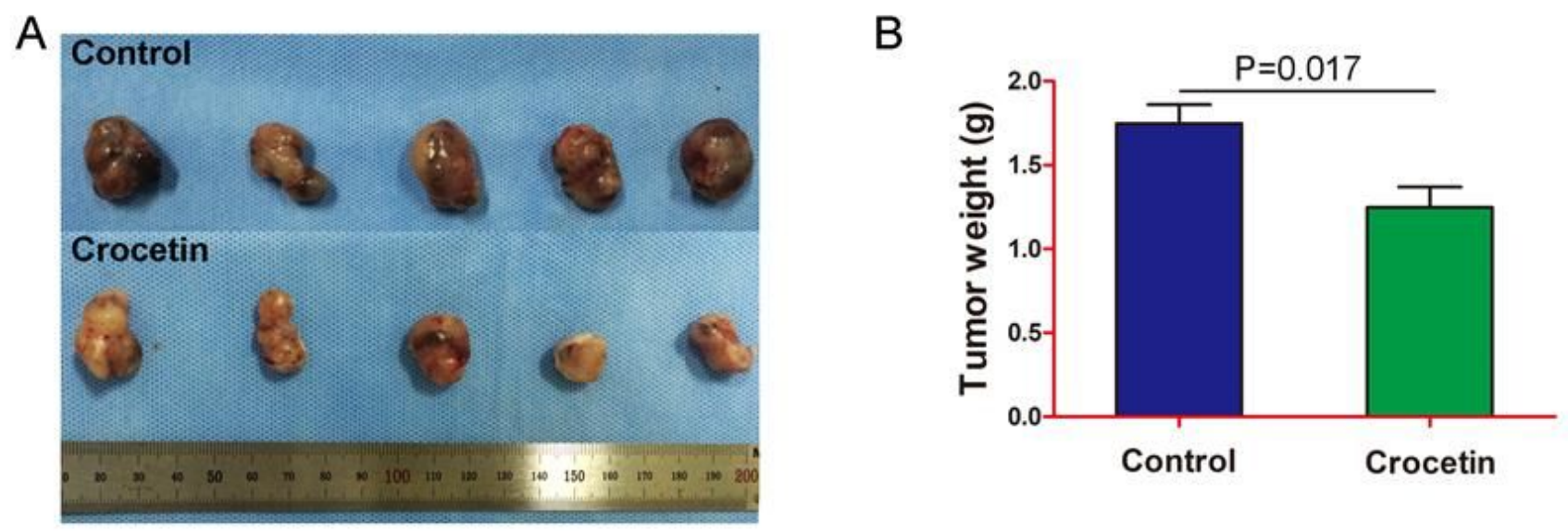

C

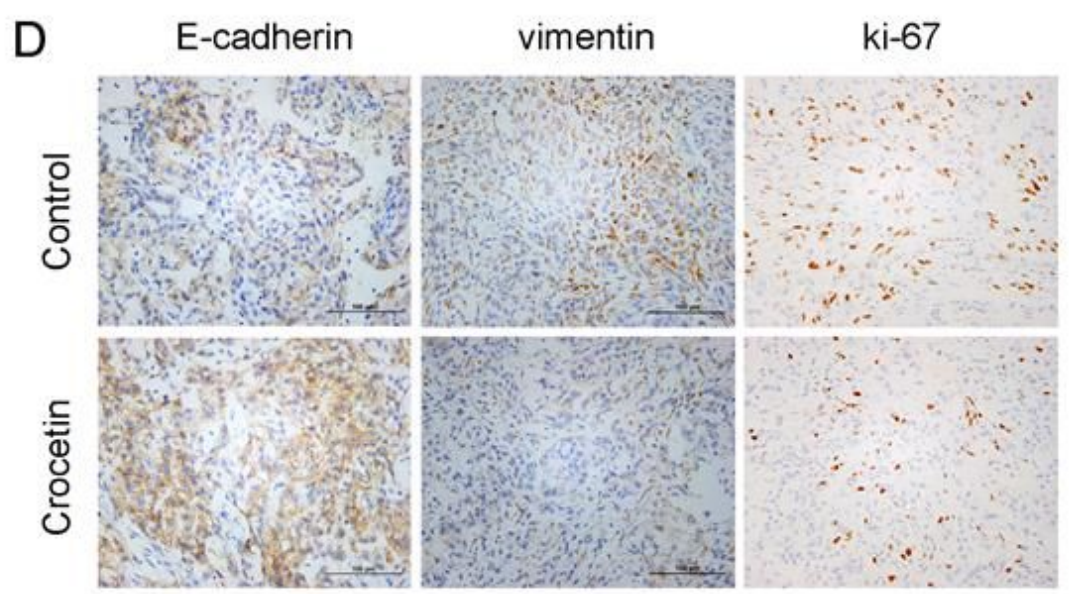

Figure 6

Effect of crocetin on tumor growth in vivo. a. Representative images of xenograft tumors formed by Hs746T GC cells treated with or without crocetin. b. Average weights of xenograft tumors in nude mice. c. Tumor volumes are measured every week. $d$. Expression of E-cadherin, vimentin, and Ki 67 in xenograft tumors (bottom right corner: $100 \mu \mathrm{m}$ ). Data are represented as mean \pm SEM of three independent experiments. Student's t-test, ${ }^{*} \mathrm{P}<0.05,{ }^{*} \mathrm{P}<0.01$. 

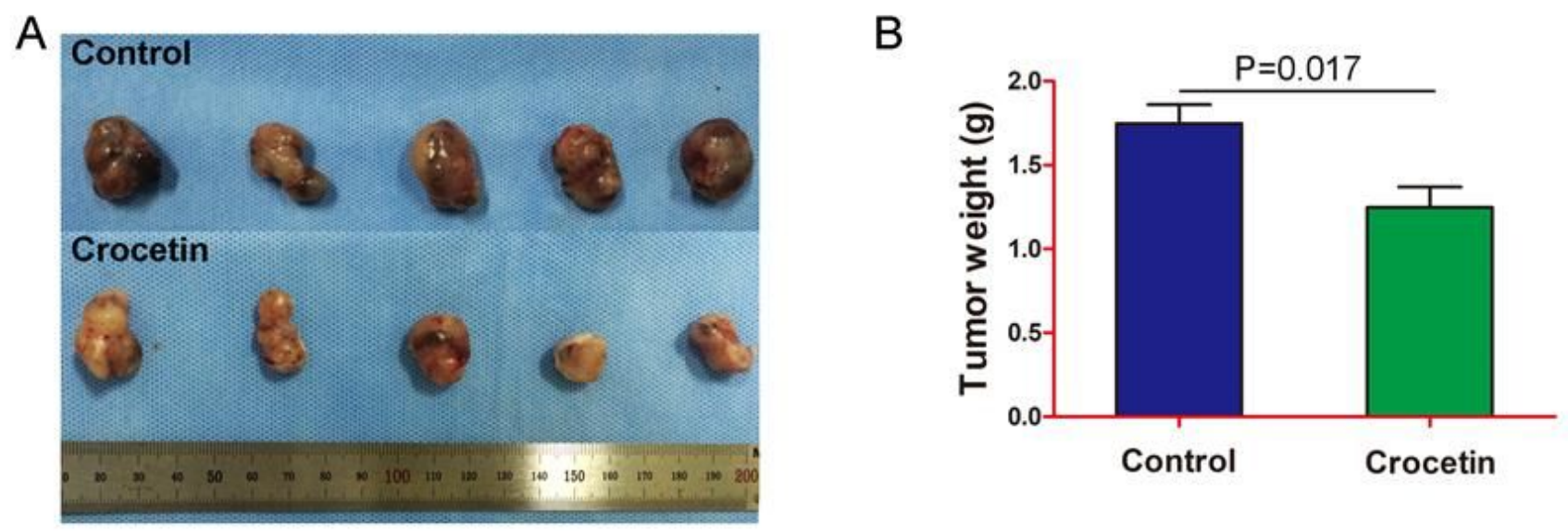

C

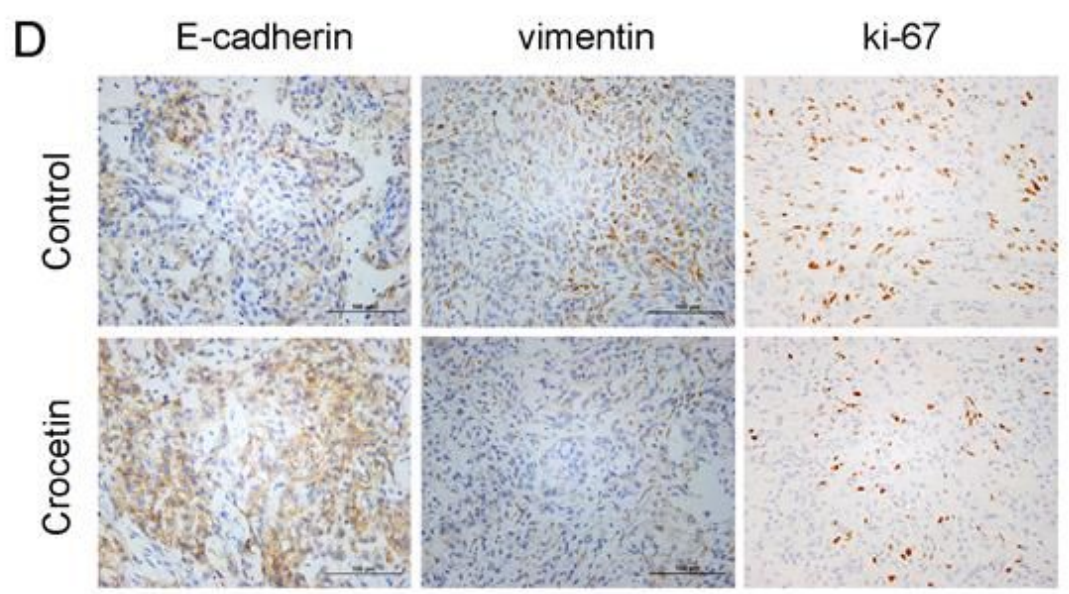

Figure 6

Effect of crocetin on tumor growth in vivo. a. Representative images of xenograft tumors formed by Hs746T GC cells treated with or without crocetin. b. Average weights of xenograft tumors in nude mice. c. Tumor volumes are measured every week. $d$. Expression of E-cadherin, vimentin, and Ki 67 in xenograft tumors (bottom right corner: $100 \mu \mathrm{m}$ ). Data are represented as mean \pm SEM of three independent experiments. Student's t-test, ${ }^{*} \mathrm{P}<0.05,{ }^{*} \mathrm{P}<0.01$. 